\title{
Berry Heterogeneity as a Possible Factor Affecting the Potential of Seed Mechanical Properties to Classify Wine Grape Varieties and Estimate Flavanol Release in Wine-like Solution
}

\author{
F. Torchio ${ }^{\dagger}$, S. Giacosa ${ }^{\dagger}$, S. Río Segade*, V. Gerbi, L. Rolle
}

Università di Torino, DISAFA - Dipartimento di Scienze Agrarie, Forestali e Alimentari, Via Leonardo da Vinci 44, 10095

Grugliasco, Italy

$\dagger$ These authors contributed equally to this study

Submitted for publication: August 2013

Accepted for publication: October 2013

Key words: Instrumental texture analysis, mechanical parameters, wine grape seeds, berry heterogeneity, varietal classification, extractable phenols prediction

\begin{abstract}
Seed mechanical properties were instrumentally measured by compression testing in thirty white and red wine grape varieties at harvest. The effect of berry heterogeneity in a vineyard on these seed texture parameters was also evaluated to improve the understanding of intra-sample variability. Furthermore, the mechanical properties of the seeds were assessed as possible predictors of their phenolic extractability. The results show that the texture parameters of the seeds are independent of the location of the berry in the vineyard and the soluble solid content at harvest. Densimetric flotation of the berries permits the reduction of the intra-sample variability that could hinder the differentiation and/or classification of wine grape varieties according to seed mechanical attributes. Cluster analysis classified the wine grape varieties studied into three groups according to seed hardness (low: 32.51 to $40.80 \mathrm{~N}$, intermediate: 42.84 to $44.99 \mathrm{~N}$, high: 46.71 to $57.78 \mathrm{~N}$ ). The relationships between the seed mechanical properties and the extractable content of phenolic compounds, determined by spectrophotometric and chromatographic reference chemical methods, were evaluated by means of correlation analysis. Linear regression calibration models were developed for each cluster. The statistical parameters highlighted that total flavonoids, proanthocyanidins and flavanols reactive to vanillin can be predicted successfully from the seed mechanical properties for the varieties having low and intermediate seed hardness (SEC\% ca. 20, RPIQ > 1.6). For varieties with harder seeds, a satisfactory predictive accuracy seems to require the construction of separate calibration models for each cultivar (Nebbiolo, SEC\% ca. 20, RPIQ > 2.2).
\end{abstract}

\section{INTRODUCTION}

The phenolic composition of the grape strongly contributes to the sensorial quality of the wine. A study performed to determine the relationship between the phenolic composition of the grapes and the projected market price of the resulting wines showed that wines in a lower price category presented lower flavanol contents (Cáceres et al., 2012). Flavanols are important phenolic compounds in the grape and wine because these compounds govern perceived quality parameters like astringency, bitterness and colour (Peleg et al., 1999; Cheynier et al., 2006; McRae \& Kennedy, 2011; ObrequeSlier et al., 2011). Grape seeds are a rich source of gallic acid and flavanols, particularly monomeric catechins (catechin, epicatechin and epicatechin-3-O-gallate), as well as their oligomeric and polymeric procyanidins (Monagas et al., 2003; Rodríguez Montealegre et al., 2006; Mattivi et al.,
2009; Ferrer-Gallego et al., 2010). Low molecular weight flavanols are important determinants of bitterness, whereas astringency increases with an increase in the chain length and galloylation percentage (Peleg et al., 1999; Cheynier et al., 2006; Ferrer-Gallego et al., 2010; Obreque-Slier et al., 2011). Otherwise, flavanols undergo complex interactions with anthocyanins during winemaking and wine ageing, and therefore play a key role in the colour of red wines (Cheynier et al., 2006; Pérez-Magariño \& González-San José, 2006; Puškaš \& Miljić, 2012).

Wine grape varieties differ widely in the qualitative and quantitative flavanolic composition of the seeds (Rodríguez Montealegre et al., 2006; Mattivi et al., 2009; Kotseridis et al., 2012; Obreque-Slier et al., 2012). This compositional diversity is of great technological relevance for the adaptation

*Corresponding author: E-mail address: susana.riosegade@unito.it [Tel.: +39 0116708558; Fax: +39 0116708549]

Aknowledgements: Part of this study was funded by the Fondazione Fojanini di Studi Superiori (Sondrio, Italy), and part by the project Ricerca \& Innovazione per il Miglioramento della Sostenibilità della Filiera Agro-alimentare ECOFOOD - Finanziamento PSR-FEASR - cofinanziamento dall'UE, dal Ministero dell'economia e delle Finanze e dalla Regione Piemonte; Pubblicazione $n^{\circ}$ 05. The authors thank Anna Schneider, for the samples collected in Piedmont, and Graziano Murada, for kindly providing the grape samples from Valtellina 
of the winemaking techniques to the oenological potential of a particular cultivar (Sacchi et al., 2005). Furthermore, the changes occurring during grape ripening affect the content and extraction of phenolic compounds in the seeds. The extractable amount of seed flavanols gradually declines with the advance of ripening because of oxidation reactions that favour the increased association of these compounds with cell-wall components (Kennedy et al., 2000a, 2000b; Downey et al., 2003; Cadot et al., 2006; Ferrer-Gallego et al., 2010; Lorrain et al., 2011; Obreque-Slier et al., 2012). In this sense, the knowledge at harvest of the flavanolic composition of the seeds that can be extracted into the wine facilitates winemaking management.

The medium integument undergoes an intensive lignification during ripening, which results in hardening of the grape seed (Cadot et al., 2006) and, therefore, the hardness has been proposed as a sensory descriptor of the seeds (Le Moigne et al., 2008). Although wine grape tasting is a wellrecognised tool that is used by many wine professionals to support harvesting decisions, the subjectivity associated with the sensory perception of this texture property and the berry heterogeneity make it difficult to discriminate between ripening stages. High data variability can be reduced by means of objective measurements based on instrumental texture analysis. The compression parameters are closely related to the extractable content of phenolic compounds during ripening, particularly in the seeds. The resistance of the seed to axial deformation is negatively correlated with the phenol extractability (Rolle et al., 2012a), and the seed break force is also intrinsically linked to the extractable content of oligomeric flavanols (Torchio et al., 2012). Nevertheless, the robustness of these correlations was low, probably due to the fact that the studies were conducted either for total phenols or on heterogeneous berries in terms of ripeness at each harvest date.

The berry heterogeneity inside the vineyard is a limiting factor for characterising grape varieties and discriminating between ripening stages because of the variability in the physicochemical characteristics. Some authors have even proposed exploiting the separation of berries from different cluster positions for the elaboration of different quality wines (Noguerol-Pato et al., 2012). Others have chosen the densimetric sorting of the berries as the best option in the winery to separate grapes with different quality parameters, this process being automatised through the use of densimetric berry-sorting machines (Rolle et al., 2012b).

In this work, thirty wine grape varieties were characterised according to the mechanical properties of the intact seeds measured during compression testing. The effect of the berry heterogeneity in a vineyard was evaluated to improve the understanding of the intra-sample variability that could influence the discriminating ability of the seed texture parameters. The densimetric sorting allowed the berries to be separated at harvest into more homogeneous groups in terms of ripeness, thereby also facilitating the study of the ripening effect on the texture attributes of the seeds. Furthermore, the performance of the instrumental mechanical parameters of grape seeds as possible predictors of the extractable phenolic composition determined by reference chemical methods was comprehensively investigated by means of the establishment of robust relationships using the varieties studied. The modelling of those strongest and most significant correlations may provide insight into the rapid estimation of the amount of phenolic compounds in grape seeds that can be extracted during winemaking.

\section{MATERIALS AND METHODS \\ Grape samples}

Whole bunches of seven white (Arneis, Chardonnay, Erbaluce, Malvasia Bianca, Moscato Bianco, Riesling Italico, Sauvignon Blanc) and 23 red (Albarossa, Avanà, Barbera, Brachetto d'Acqui, Cabernet Sauvignon, Calabrese, Cinsault, Croatina, Dolcetto, Freisa, Grenache, Grignolino, Malvasia di Schierano, Merlot, Mourvèdre, Nebbiolo, Petit Rouge, Petit Verdot, Pignolo Spano, Pinot Noir, Ruchè, Sangiovese, Syrah) grape cultivars (Vitis vinifera L.) were harvested at technological maturity from the same vineyard located in Grinzane Cavour (Piedmont, Cuneo Province, north-west Italy) in 2010. Furthermore, grape samples of the Nebbiolo red cultivar were collected at two different ripening stages in ten commercial vineyards located in Valtellina (Sondrio Province, Lombardy, northern Italy) in 2010 and 2011. The study was performed separately for each wine grape cultivar, harvest date and vineyard. Bunches $(\mathrm{n}=10)$ were randomly collected from ten vines (one bunch per vine) selected to ensure a representative sampling. Once in the laboratory, a subsample of approximately $1.5 \mathrm{~kg}$ of grapes (ca. 1000 to 1200 berries) was randomly selected by picking berries from different positions in the cluster (shoulders, middle and bottom). For each subsample, one set of 100 berries was randomly selected and used for determining the technological ripeness parameters in the grape must obtained by manual crushing and centrifugation at $3000 \times \mathrm{g}$ for 10 min at $20^{\circ} \mathrm{C}$ (Universal $32 \mathrm{R}$, Hettich, Tuttlingen, Germany).

For the estimation of the variability in the instrumental texture parameters of the seeds within and among clusters, the seeds $(n=5)$ of Pinot Noir berries located in a given position of the cluster were analysed separately from those of the berries located in the other two positions, with a total of 15 seeds per cluster (Torchio et al., 2012). The study of the effect of berry heterogeneity on the mechanical properties of the seeds also required an analysis of all the seeds belonging to the same berry for one set of 10 Pinot Noir berries randomly sampled. Afterwards, in order to better define the different ripening states present at the same harvest date and to improve intra-sample homogeneity, the remaining Pinot Noir berries were sorted according to their density by flotation using different saline solutions (from 100 to $170 \mathrm{~g} / \mathrm{L}$ sodium chloride) and following the protocol described by Rolle et al. (2011). The berry density classes selected were: $\mathrm{A}=1075 \mathrm{~kg} / \mathrm{m}^{3}, \mathrm{~B}=1081 \mathrm{~kg} / \mathrm{m}^{3}, \mathrm{C}=1088 \mathrm{~kg} / \mathrm{m}^{3}$ and $\mathrm{D}=$ $1094 \mathrm{~kg} / \mathrm{m}^{3}$. For each density class, a subsample of 50 sorted berries was used to evaluate the effect of the density class on the mechanical properties of the seeds $(n=50)$. An average subsample of 50 unsorted berries (UnS) was also used with the same last aim, with the total number of seeds analysed being 250 .

The remaining berries of the other 29 wine grape varieties were also sorted densimetrically as mentioned above, with the exception of the Nebbiolo cultivar from the Valtellina 
growing zone. In this case, the selected berry density classes were: $\mathrm{D}=1094 \mathrm{~kg} / \mathrm{m}^{3}, \mathrm{E}=1100 \mathrm{~kg} / \mathrm{m}^{3}$ and $\mathrm{F}=1107 \mathrm{~kg} / \mathrm{m}^{3}$, with the exception of the Cinsault and Sangiovese cultivars, in which density class $\mathrm{C}\left(1088 \mathrm{~kg} / \mathrm{m}^{3}\right)$ predominated over the F. The criterion was to select berries with three ripeness grades that corresponded to the most representative values of probable alcohol $(12.5,13.5$ and $14.5 \% \mathrm{v} / \mathrm{v}$, respectively). The berries in each density class were weighed and the distribution percentage was established. For both variety and density class (or harvest date and vineyard for the Nebbiolo from Valtellina), one set of 30 sorted berries was randomly selected and subdivided into three replicates of 10 grape berries that were weighed with a technical balance (Gibertini E1700, Modena, Italy). Subsequently, the three replicates of 10 berry seeds obtained were also weighed. The intact seeds of each replicate were individually compressed and quickly immersed in an extracting wine-like solution. In all studies, unless stated otherwise, only one randomly selected seed per berry was used for analysis. These were previously separated from the pulp and carefully cleaned with adsorbent paper. This permitted a wider variation range with the same number of seeds to be covered, facilitating the characterisation of wine grape varieties according to the mechanical properties of the seeds, the evaluation of the ripening effect on these texture parameters, and the prediction of extractable phenolic compounds of the seeds, determined by reference chemical methods, from the mechanical attributes.

\section{Instrumental mechanical properties}

The mechanical properties were determined directly on the intact seeds by a compression test. Each seed was individually compressed at $1 \mathrm{~mm} / \mathrm{s}$ speed under $50 \%$ deformation using a TA.XT2i Plus Texture Analyzer (Stable Micro Systems, Surrey, UK) equipped with an SMS HDP/90 platform, an SMS P/35 probe and a $50 \mathrm{~kg}$ load cell (Torchio et al., 2012). The following instrumental mechanical parameters were determined: the seed break force $\left(\mathrm{N}\right.$, as $\left.\mathrm{F}_{\mathrm{s}}\right)$, the seed break energy $\left(\mathrm{mJ}\right.$, as $\left.\mathrm{W}_{\mathrm{s}}\right)$, the resistance of the seed to the axial deformation $\left(\mathrm{N} / \mathrm{mm}\right.$, as $\left.\mathrm{E}_{\mathrm{s}}\right)$, and the seed deformation index (\%, as DI ) (Rolle et al., 2012c). This last index was calculated as the distance of the seed break point/seed height $\times 100$. Before each test session, the instrument was calibrated for force and distance.

\section{Chemical analysis}

Solvents of HPLC gradient grade and all other chemicals of analytical reagent grade were purchased from Sigma (Milan, Italy). The solutions were prepared in deionised water produced by a Purelab Classic system (Elga Labwater, Marlow, United Kingdom). Of the phenol standards, gallic acid (GA), (+)-catechin (CA), (-)-epicatechin (EC) and (-)-epicatechin gallate (ECG) were obtained from Sigma, and cyanidin chloride and procyanidins $B_{1}$ and $B_{2}$ were purchased from Extrasynthèse (Genay, France).

Technological ripeness parameters. The concentration of total soluble solids ( ${ }^{\circ}$ Brix, as SSC) was measured with an Atago 0 to $32^{\circ}$ Brix temperature-compensating refractometer (Atago Corporation, Tokyo, Japan), and the $\mathrm{pH}$ was determined by potentiometry using a Crison electrode (Carpi,
Italy). Titratable acidity (TA), expressed as $\mathrm{g} / \mathrm{L}$ of tartaric acid, was estimated using the method of the International Organisation of Vine and Wine (OIV, 2008). Malic acid and tartaric acid were quantified $(\mathrm{g} / \mathrm{L})$ using a P100-AS3000 HPLC system (Thermo Electron Corporation, Waltham, MA, USA) equipped with a UV detector (UV3000) set to 210 $\mathrm{nm}$. The analyses were performed according to the method proposed by Giordano et al. (2009). The data analysis was carried out using the ChromQuest chromatography data system (ThermoQuest, Inc., San Jose, CA, USA).

Extraction and determination of seed phenols. In the reference method, each replicate of 10 berry seeds previously compressed was immediately immersed in $10 \mathrm{~mL}$ of a winelike solution and maintained at $25^{\circ} \mathrm{C}$ for seven days (Torchio et al., 2012). This wine-like hydroalcoholic solution consisted of ethanol/water $(12 / 88 \mathrm{v} / \mathrm{v})$ containing $2 \mathrm{~g} / \mathrm{L} \mathrm{Na}_{2} \mathrm{~S}_{2} \mathrm{O}_{5}$ (to avoid the possible oxidation of phenolic compounds) and $5 \mathrm{~g} / \mathrm{L}$ tartaric acid, which was buffered at $\mathrm{pH} 3.2$ using $\mathrm{NaOH} 0.1 \mathrm{~N}$. The extracts were filtered through a $0.20 \mu \mathrm{m}$ filter, bottled and stored at $4^{\circ} \mathrm{C}$ until they were analysed. Spectrophotometric methods were used to determine absorbance at $280 \mathrm{~nm}\left(\right.$ as $\left.\mathrm{A}_{280}\right)$ and the extractable content of total flavonoids $[\mathrm{mg}(+)$-catechin/kg grape or $\mathrm{mg} / \mathrm{g}$ seed, as $\mathrm{TF}$, proanthocyanidins (viz. polymeric flavanols, expressed as $\mathrm{mg}$ cyanidin chloride/ $\mathrm{kg}$ grape or $\mathrm{mg} / \mathrm{g}$ seed, as PRO) and flavanols reactive to vanillin [viz. oligomeric flavanols, expressed as $\mathrm{mg}(+)$-catechin/kg grape or $\mathrm{mg} / \mathrm{g}$ seed, as FRV] (Di Stefano \& Cravero, 1991; Torchio et al., 2012). Proanthocyanidins were determined after acid hydrolysis with warming (Bate-Smith reaction), using a ferrous salt $\left(\mathrm{FeSO}_{4}\right)$ as catalyst. A UV-1800 spectrophotometer (Shimazdu Corporation, Kyoto, Japan) was used. The relative standard deviations of phenolic compound determinations, based on repeated analyses $(n=20)$ of 10 sample extracts, were 0.93 , 1.74 and $2.80 \%$ for TF, PRO and FRV, respectively (Torchio et al., 2010).

The determination of gallic acid and individual monomeric and dimeric flavanols was performed according to a method adapted from Downey et al. (2003). An Agilent 1260 Infinity HPLC system (Milford, MA, USA) equipped with a diode array detector (DAD) was used. The chromatographic separation was carried out at $25^{\circ} \mathrm{C}$ on a LiChroCART 250-2 Purospher STAR RP-18 analytical column $(5 \mu \mathrm{m}, 250 \mathrm{~mm} \times 2 \mathrm{~mm}$ i.d.) purchased from Merck (Darmstadt, Germany). The injection volume was $20 \mu \mathrm{L}$. The mobile phases consisted of $0.2 \%$ aqueous phosphoric acid (mobile phase A) and a mixture of acetonitrile/ $0.2 \%$ phosphoric acid (4:1) (mobile phase B) at a flow rate of 0.2 $\mathrm{mL} / \mathrm{min}$. The two mobile phases were filtered through a 0.20 $\mu \mathrm{m}$ filter. A linear gradient was used for the separation of flavanols, starting at $0 \% \mathrm{~B}$ to $10 \% \mathrm{~B}$ in $5 \mathrm{~min}$, maintaining at $10 \% \mathrm{~B}$ for $35 \mathrm{~min}$, increasing to $17 \% \mathrm{~B}$ in $15 \mathrm{~min}$, to $19 \% \mathrm{~B}$ in $10 \mathrm{~min}$, and maintaining at $19 \% \mathrm{~B}$ for $10 \mathrm{~min}$. The column was then washed with $100 \% \mathrm{~B}$ for $10 \mathrm{~min}$ and equilibrated for $10 \mathrm{~min}$ prior to each analysis. The UV-VIS spectra were acquired from 230 to $400 \mathrm{~nm}$, and the detection wavelength was set at $280 \mathrm{~nm}$. The identification was achieved by comparing the absorption spectra and retention times with those of pure standards. The quantification $(\mathrm{mg} / \mathrm{kg}$ grape or 
$\mathrm{mg} / \mathrm{g}$ seed) was carried out by the external standard method. All analyses were performed in triplicate.

\section{Statistical analysis}

Statistical analyses were carried out using the SPSS software package version 19.0 (SPSS Inc., Chicago, IL, USA). Oneway analysis of variance (ANOVA) was used to establish significant differences. A cluster analysis was performed to classify wine grape varieties according to the mechanical properties of the seeds, using the average linkage between groups and squared Euclidean distance. Pearson's correlation coefficients were calculated to determine significant relationships between the instrumental texture parameters and the phenolic composition of the seeds. For prediction purposes, the sample set was randomly subdivided into the calibration set (about 2/3) and the validation set (about 1/3), the two sets being associated with comparable ranges of phenolic compounds. The performance of calibration models developed by regression analysis was assessed from the correlation coefficient of calibration $\left(\mathrm{R}_{\mathrm{c}}\right)$ and the standard error of calibration (SEC). The standard error of calibration was also standardised (SEC\%) by rating its value to the mean of the calibration population, and it is related to the mean error of the model. On the other hand, the predictive accuracy of the calibration models was evaluated from the correlation coefficient of validation $\left(\mathrm{R}_{\mathrm{v}}\right)$ and the standard error of prediction (SEP). Furthermore, the coefficient of variation $(\mathrm{SEP} \%)$ was calculated as the ratio of the SEP value to the mean of the validation population. Other indices evaluated with this objective were the residual predictive deviation (RPD) and the residual predictive interquartile amplitude (RPIQ). The first statistical index is the most commonly used to account for model reliability and was defined as the ratio between the standard deviation (SD) of the validation set and the SEP value. The other is based on quartiles and was calculated as the ratio of the interquartile amplitude of the validation population to the SEP value (Bellon-Maurel et al., 2010).

\section{RESULTS AND DISCUSSION}

Effect of berry heterogeneity on seed mechanical properties

A preliminary study was performed on the Pinot Noir cultivar to assess if the berry heterogeneity typically present in a vineyard influences the variability in the mechanical properties of the seeds at harvest. The differences in the texture parameters of the seed tissues were not significant $(p>0.05)$ within the same berry or among grape berries randomly sampled. On the other hand, the mechanical attributes also agreed for the seeds belonging to berries from different positions within the cluster $(p>0.05)$. This confirms the results reported in another work, where no influence of the position of the grape berry within the cluster was observed on the mechanical properties of the seeds (Torchio et al., 2012). For most of the clusters analysed, the variability (as relative standard deviation) was higher in the middle position than in the shoulder and bottom positions. The mechanical attributes of the seeds agreed statistically for berries from different clusters $(p>0.05)$. After verifying that the location of the berry in the vineyard was not an influencing factor on the texture parameters of the seeds, the study was completed with the evaluation of the berry density effect on these parameters at the harvest date. There were no significant differences $(p>0.05)$ in the mechanical parameters of the seeds from berries belonging to the four density classes studied. Figure 1 shows that the highest variability corresponded to the average subsample (unsorted berries), whereas the ripest berries (density classes C and D) had the lowest variability in all of the mechanical properties of the seeds, although this behaviour was not as evident for the seed deformation index. The variation within the average subsample was even higher than that found among clusters.

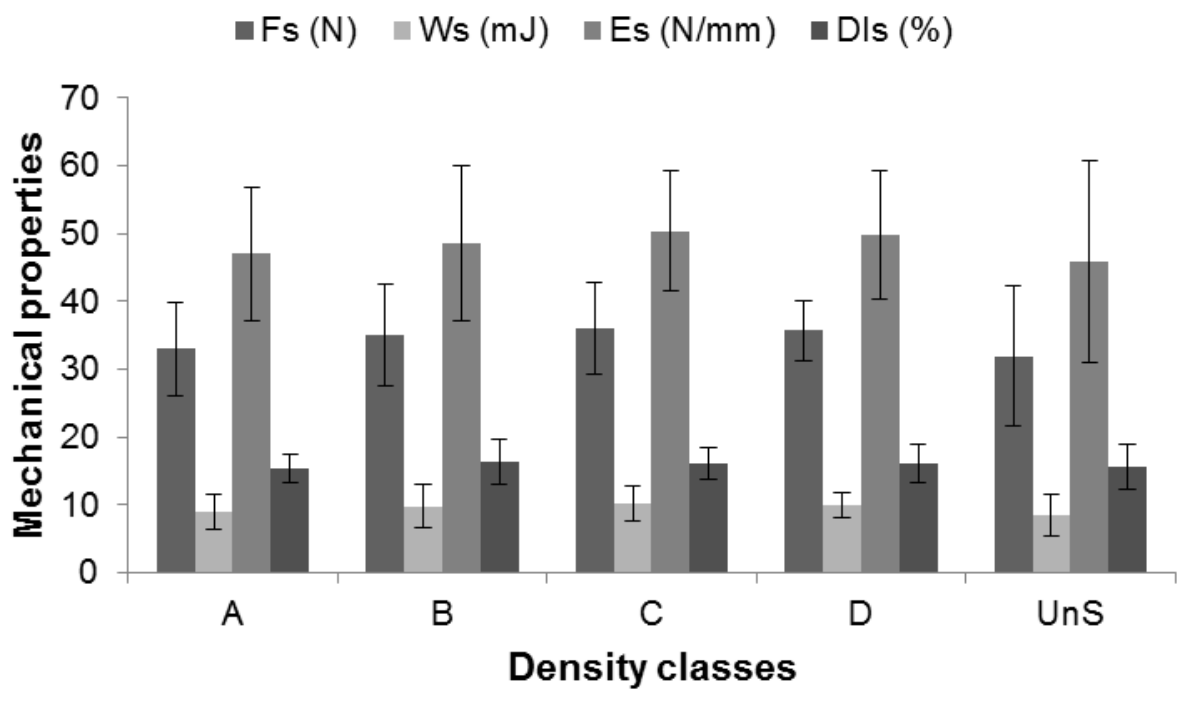

FIGURE 1

Instrumental mechanical properties of Pinot Noir seeds from densimetrically sorted grape berries at harvest. A $=1075 \mathrm{~kg} / \mathrm{m}^{3}$, $\mathrm{B}=1081 \mathrm{~kg} / \mathrm{m}^{3}, \mathrm{C}=1088 \mathrm{~kg} / \mathrm{m}^{3}, \mathrm{D}=1094 \mathrm{~kg} / \mathrm{m}^{3}, \mathrm{UnS}=$ unsorted. $\mathrm{F}_{\mathrm{s}}=$ seed break force, $\mathrm{W}_{\mathrm{s}}=$ seed break energy, $\mathrm{E}_{\mathrm{s}}=$ resistance of the seed to the axial deformation, $\mathrm{DI}_{\mathrm{s}}=$ seed deformation index. 
Therefore, the densimetric sorting of the berries permits the reduction of the intrasample variability that could hinder the differentiation and/or classification of wine grape varieties according to the mechanical attributes of the seeds.

\section{Effect of ripening on seed mechanical properties}

Table 1 shows the distribution percentage of the berries in four density classes and average technological ripeness parameters at harvest for a total of thirty wine grape varieties. For each variety, berries with different total soluble solids contents (SSC) are present in a vineyard because of a heterogeneous ripening process. Furthermore, the distribution percentage depended mainly on the variety. In fact, varieties with the same value of SSC had different distribution percentages, and no other technological ripeness parameter seemed to be a determining factor in this distribution. The average SSC at harvest ranged from 19.5 to $25.4{ }^{\circ}$ Brix, corresponding to the Syrah and Avaná cultivars, respectively. Within each density class there were differences in the SSC values among varieties with variation ranges of 17.9 to $21.4,18.7$ to 23.5 , 21.8 to 24.9 and 21.4 to $25.4{ }^{\circ}$ Brix for the density classes C, D, E and F, respectively. This could be due to the size effect on berry density. Berries with the same SSC can have different sizes and therefore would belong to different density classes. The sugar concentration is directly linked to berry size and berry fresh weight by means of a negative correlation (Dai et al., 2009). These last two parameters also showed an inverse trend with berry density (Rolle et al., $2012 b$ ). Therefore, it was necessary to study the effect of berry maturity in terms of density on the texture parameters of

TABLE 1

Distribution percentage of berries in four density classes and average technological ripeness parameters at harvest.

\begin{tabular}{|c|c|c|c|c|c|c|c|c|c|c|}
\hline \multirow{2}{*}{ Grape variety } & \multirow[b]{2}{*}{ Colour } & \multicolumn{4}{|c|}{$\begin{array}{l}\text { Berries distribution in density } \\
\text { classes }\end{array}$} & \multicolumn{5}{|c|}{ Technological ripeness parameters } \\
\hline & & $\mathrm{C}(\%)$ & $\mathrm{D}(\%)$ & $\mathrm{E}(\%)$ & $\mathrm{F}(\%)$ & $\begin{array}{c}\mathrm{SSC} \\
\left({ }^{\mathrm{o}} \text { Brix }\right)\end{array}$ & $\begin{array}{c}\mathrm{TA}(\mathrm{g} / \mathrm{L} \\
\text { tartaric acid })\end{array}$ & $\mathrm{pH}$ & $\begin{array}{c}\text { Malic } \\
\text { acid }(\mathrm{g} / \mathrm{L})\end{array}$ & $\begin{array}{c}\text { Tartaric } \\
\operatorname{acid}(\mathrm{g} / \mathrm{L})\end{array}$ \\
\hline Albarossa & $\mathrm{R}$ & 5.1 & 30.7 & 26.3 & 28.7 & 23.6 & 10.72 & 2.87 & 2.6 & 10.0 \\
\hline Arneis & $\mathrm{W}$ & 11.5 & 17.0 & 10.1 & 37.2 & 23.1 & 6.56 & 3.10 & 1.7 & 7.1 \\
\hline Avaná & $\mathrm{R}$ & 10.9 & 8.7 & 9.0 & 32.2 & 25.4 & 5.55 & 3.24 & 1.4 & 7.3 \\
\hline Barbera & $\mathrm{R}$ & 17.9 & 8.3 & 8.7 & 25.4 & 24.3 & 10.59 & 2.96 & 2.8 & 8.9 \\
\hline Brachetto d'Acqui & $\mathrm{R}$ & 3.1 & 22.9 & 28.0 & 34.6 & 22.6 & 8.84 & 3.11 & 3.8 & 7.4 \\
\hline Cabernet Sauvignon & $\mathrm{R}$ & 13.0 & 27.8 & 42.8 & 16.4 & 22.6 & 6.43 & 3.19 & 2.1 & 6.8 \\
\hline Calabrese & $\mathrm{R}$ & 49.1 & 38.5 & 7.6 & 4.9 & 21.0 & 7.99 & 3.02 & 2.5 & 7.8 \\
\hline Chardonnay & W & 16.2 & 36.2 & 36.7 & 6.3 & 22.6 & 7.40 & 3.24 & 2.6 & 6.9 \\
\hline Cinsault & $\mathrm{R}$ & 21.6 & 51.8 & 21.6 & 1.7 & 22.7 & 4.98 & 3.24 & 2.4 & 5.7 \\
\hline Croatina & $\mathrm{R}$ & 22.3 & 11.1 & 33.0 & 28.0 & 22.2 & 6.45 & 3.19 & 2.4 & 6.6 \\
\hline Dolcetto & $\mathrm{R}$ & 43.0 & 24.9 & 17.6 & 11.8 & 20.5 & 6.24 & 3.30 & 2.4 & 6.8 \\
\hline Erbaluce & W & 32.9 & 35.7 & 17.7 & 10.6 & 21.9 & 7.14 & 3.08 & 2.1 & 7.6 \\
\hline Freisa & $\mathrm{R}$ & 0.8 & 9.7 & 43.1 & 38.2 & 22.8 & 8.62 & 3.15 & 3.4 & 6.9 \\
\hline Grenache & $\mathrm{R}$ & 4.3 & 15.1 & 28.8 & 21.4 & 23.6 & 5.49 & 3.25 & 2.0 & 5.9 \\
\hline Grignolino & $\mathrm{R}$ & 11.5 & 11.0 & 37.5 & 26.7 & 22.5 & 6.84 & 3.20 & 2.7 & 6.3 \\
\hline Malvasia Bianca & W & 46.1 & 12.7 & 24.0 & 16.3 & 20.8 & 6.54 & 3.19 & 2.8 & 6.2 \\
\hline Malvasia di Schierano & $\mathrm{R}$ & 22.4 & 35.3 & 30.6 & 9.1 & 21.7 & 6.00 & 3.17 & 2.3 & 6.3 \\
\hline Merlot & $\mathrm{R}$ & 3.7 & 34.2 & 39.4 & 20.2 & 23.2 & 5.55 & 3.47 & 2.4 & 6.6 \\
\hline Moscato Bianco & W & 19.2 & 14.0 & 24.8 & 25.4 & 22.4 & 7.21 & 3.18 & 2.7 & 7.0 \\
\hline Mourvèdre & $\mathrm{R}$ & 43.2 & 35.6 & 15.1 & 3.9 & 21.1 & 7.15 & 3.28 & 4.1 & 5.5 \\
\hline Nebbiolo & $\mathrm{R}$ & 1.6 & 16.0 & 65.5 & 16.3 & 23.3 & 8.50 & 2.96 & 2.2 & 8.2 \\
\hline Petit Rouge & $\mathrm{R}$ & 11.4 & 18.6 & 24.8 & 31.8 & 24.1 & 6.08 & 3.23 & 2.2 & 6.7 \\
\hline Petit Verdot & $\mathrm{R}$ & 0.0 & 14.3 & 38.6 & 45.2 & 22.4 & 8.85 & 3.19 & 4.5 & 6.4 \\
\hline Pignolo Spano & $\mathrm{R}$ & 5.6 & 18.9 & 35.7 & 19.0 & 22.6 & 5.72 & 3.24 & 2.4 & 6.1 \\
\hline Pinot Noir & $\mathrm{R}$ & 9.6 & 23.9 & 34.4 & 22.0 & 23.1 & 7.32 & 3.22 & 2.8 & 6.7 \\
\hline Riesling Italico & W & 33.1 & 29.7 & 15.7 & 11.7 & 22.6 & 6.12 & 3.18 & 1.6 & 7.6 \\
\hline Ruchè & $\mathrm{R}$ & 13.8 & 21.7 & 29.4 & 24.3 & 21.8 & 7.04 & 3.21 & 2.7 & 6.9 \\
\hline Sangiovese & $\mathrm{R}$ & 27.2 & 47.4 & 15.1 & 0.0 & 21.6 & 7.55 & 3.08 & 2.7 & 7.1 \\
\hline Sauvignon Blanc & $\mathrm{W}$ & 4.1 & 27.8 & 33.2 & 30.9 & 23.6 & 8.79 & 3.04 & 2.4 & 8.5 \\
\hline Syrah & $\mathrm{R}$ & 71.6 & 19.2 & 6.3 & 2.8 & 19.5 & 6.98 & 3.19 & 3.4 & 6.1 \\
\hline
\end{tabular}

$\mathrm{SSC}=$ total soluble solids content, $\mathrm{TA}=$ titratable acidity. $\mathrm{R}=\mathrm{red}, \mathrm{W}=$ white. $\mathrm{C}=1088 \mathrm{~kg} / \mathrm{m}^{3}, \mathrm{D}=1094 \mathrm{~kg} / \mathrm{m}^{3}, \mathrm{E}=1100$ $\mathrm{kg} / \mathrm{m}^{3}, \mathrm{~F}=1107 \mathrm{~kg} / \mathrm{m}^{3}$. 
the seeds for wine grape varieties with different distribution percentages of the berries in density classes. As can be seen in Table 2, the mechanical properties of the seeds were not significantly influenced by the berry density at harvest for most of the varieties. Furthermore, the few significant differences found did not show a clear trend for the different varieties studied. Despite the intensive lignification of the tissues during ripening that involved seed hardening (Cadot et al., 2006), some works have also reported no significant change in the mechanical attributes of berry seeds $\left(\mathrm{F}_{\mathrm{s}}, \mathrm{W}_{\mathrm{s}}, \mathrm{E}_{\mathrm{s}}\right.$ and $\mathrm{DI}_{\mathrm{s}}$ ) for densimetrically sorted Barbera grapes harvested at the same date (Torchio et al., 2010). In contrast, significant differences were found in the compression parameters of Cabernet Sauvignon seeds $\left(\mathrm{W}_{\mathrm{s}}, \mathrm{E}_{\mathrm{s}}\right.$ and $\left.\mathrm{DI}_{\mathrm{s}}\right)$ when the ripening effect was studied for several weeks (Rolle et al., 2012d). Nevertheless, the changes occurred only in seed stiffness $\left(\mathrm{E}_{\mathrm{s}}\right)$ for sorted Cabernet Sauvignon grapes sampled at different harvest dates, even if one density class per sampling date was selected to emphasise the physiological differences among grape ripening stages (Rolle et al., 2012a). Some researchers also noted that most of the texture parameters of the seeds became steady three weeks after the end of véraison (Letaief et al., 2013). During the on-vine dehydration process of Mondeuse grapes, stability in the values of seed mechanical properties was reached after the $75^{\text {th }}$ withering day (Rolle et al., 2009). Therefore, the sampling date is a key factor in the visualisation of the ripening effect on the instrumental texture parameters of the seeds. On the other hand, the discriminating ability of the mechanical properties of the seeds may depend on the operative conditions used during the compression test (Torchio et al., 2012).

\section{Characterisation and differentiation of wine grape} varieties according to seed hardness

After verifying that the mechanical properties of the seeds were not influenced by the berry heterogeneity present in the vineyard at the harvest date, the potential of the instrumental texture parameters of seed tissues as varietal markers was evaluated. The statistical analysis of average data for the three density classes in each wine grape cultivar revealed that the seed break force $\left(\mathrm{F}_{\mathrm{s}}\right)$ was the best varietal marker, and that it was correlated significantly with the seed break energy $\left(\mathrm{W}_{\mathrm{s}}\right)$, the resistance of the seed to the axial deformation $\left(\mathrm{E}_{\mathrm{s}}\right)$ and the seed deformation index (DI $)$, with Pearson's correlation

TABLE 2

Instrumental mechanical properties of seeds from densimetrically sorted berries at harvest.

\begin{tabular}{|c|c|c|c|c|c|}
\hline Grape variety & Density class & $\mathrm{F}_{\mathrm{s}}(\mathrm{N})$ & $\mathrm{W}_{\mathrm{s}}(\mathrm{mJ})$ & $\mathrm{E}_{\mathrm{s}}(\mathrm{N} / \mathrm{mm})$ & $\mathrm{DI}_{\mathrm{s}}(\%)$ \\
\hline \multirow{3}{*}{ Albarossa } & $\mathrm{D}$ & $36.88 \pm 2.58$ & $9.81 \pm 0.63$ & $62.16 \pm 7.95$ & $14.78 \pm 0.72$ \\
\hline & $\mathrm{E}$ & $35.54 \pm 2.92$ & $8.97 \pm 1.07$ & $62.81 \pm 2.08$ & $14.15 \pm 0.34$ \\
\hline & $\mathrm{F}$ & $32.15 \pm 2.82$ & $7.86 \pm 1.37$ & $58.98 \pm 1.79$ & $13.62 \pm 0.88$ \\
\hline Sign $^{a}$ & & ns & ns & ns & ns \\
\hline \multirow{3}{*}{ Arneis } & $\mathrm{D}$ & $54.28 \pm 0.78 b$ & $13.91 \pm 0.25 b$ & $90.95 \pm 3.58$ & $14.37 \pm 0.52$ \\
\hline & $\mathrm{E}$ & $54.88 \pm 2.58 b$ & $13.87 \pm 0.08 b$ & $97.72 \pm 10.17$ & $13.98 \pm 1.00$ \\
\hline & $\mathrm{F}$ & $48.45 \pm 1.91 \mathrm{a}$ & $11.76 \pm 0.60 \mathrm{a}$ & $93.11 \pm 9.29$ & $12.92 \pm 1.05$ \\
\hline Sign & & $*$ & $* * *$ & $\mathrm{~ns}$ & $\mathrm{~ns}$ \\
\hline \multirow{3}{*}{ Avaná } & $\mathrm{D}$ & $49.50 \pm 3.98 b$ & $14.32 \pm 1.21$ & $74.25 \pm 7.12 b$ & $15.14 \pm 0.43$ \\
\hline & $\mathrm{E}$ & $36.52 \pm 7.58 \mathrm{a}$ & $11.67 \pm 1.88$ & $51.58 \pm 12.39 a$ & $16.20 \pm 1.61$ \\
\hline & $\mathrm{F}$ & $47.00 \pm 4.11 \mathrm{ab}$ & $13.77 \pm 1.34$ & $70.48 \pm 6.78 \mathrm{ab}$ & $15.56 \pm 0.34$ \\
\hline Sign & & $*$ & ns & $*$ & ns \\
\hline \multirow{3}{*}{ Barbera } & $\mathrm{D}$ & $45.28 \pm 3.84$ & $11.59 \pm 0.94$ & $80.03 \pm 9.03$ & $14.37 \pm 0.94$ \\
\hline & $\mathrm{E}$ & $44.35 \pm 2.40$ & $10.64 \pm 0.44$ & $81.20 \pm 4.83$ & $14.53 \pm 0.68$ \\
\hline & $\mathrm{F}$ & $44.24 \pm 2.97$ & $10.48 \pm 1.44$ & $86.20 \pm 0.83$ & $14.00 \pm 1.45$ \\
\hline Sign & & ns & ns & $\mathrm{ns}$ & $\mathrm{ns}$ \\
\hline \multirow{3}{*}{ Brachetto d'Acqui } & $\mathrm{D}$ & $40.43 \pm 0.79 \mathrm{ab}$ & $11.42 \pm 0.29$ & $62.67 \pm 0.36$ & $15.53 \pm 0.61$ \\
\hline & $\mathrm{E}$ & $42.46 \pm 1.68 b$ & $12.49 \pm 1.67$ & $69.50 \pm 7.36$ & $15.15 \pm 1.82$ \\
\hline & $\mathrm{F}$ & $38.63 \pm 1.88 \mathrm{a}$ & $10.70 \pm 1.58$ & $67.03 \pm 3.41$ & $13.84 \pm 1.73$ \\
\hline Sign & & $*$ & $\mathrm{~ns}$ & ns & ns \\
\hline \multirow{3}{*}{ Cabernet Sauvignon } & $\mathrm{D}$ & $42.38 \pm 1.85$ & $10.88 \pm 1.28$ & $72.66 \pm 2.95$ & $15.46 \pm 1.21$ \\
\hline & $\mathrm{E}$ & $44.91 \pm 1.31$ & $11.59 \pm 0.39$ & $77.33 \pm 1.72$ & $15.39 \pm 0.29$ \\
\hline & $\mathrm{F}$ & $41.25 \pm 3.19$ & $10.24 \pm 1.14$ & $74.32 \pm 2.06$ & $14.28 \pm 0.81$ \\
\hline Sign & & ns & ns & $\mathrm{ns}$ & ns \\
\hline \multirow{3}{*}{ Calabrese } & $\mathrm{D}$ & $50.86 \pm 9.37$ & $12.61 \pm 2.50$ & $82.98 \pm 8.52$ & $14.83 \pm 0.74$ \\
\hline & $\mathrm{E}$ & $49.86 \pm 0.50$ & $13.07 \pm 0.05$ & $79.17 \pm 2.00$ & $15.39 \pm 0.26$ \\
\hline & $\mathrm{F}$ & $49.07 \pm 2.45$ & $12.12 \pm 1.01$ & $84.39 \pm 4.37$ & $14.72 \pm 1.54$ \\
\hline
\end{tabular}


TABLE 2 (CONTINUED)

\begin{tabular}{|c|c|c|c|c|c|}
\hline Grape variety & Density class & $\mathrm{F}_{\mathrm{s}}(\mathrm{N})$ & $\mathrm{W}_{\mathrm{s}}(\mathrm{mJ})$ & $\mathrm{E}_{\mathrm{s}}(\mathrm{N} / \mathrm{mm})$ & $\mathrm{DI}_{\mathrm{s}}(\%)$ \\
\hline \multirow{3}{*}{ Chardonnay } & $\mathrm{D}$ & $37.13 \pm 1.63$ & $10.07 \pm 0.49$ & $61.46 \pm 2.51$ & $14.80 \pm 0.25 b$ \\
\hline & $\mathrm{E}$ & $36.41 \pm 1.62$ & $9.63 \pm 0.65$ & $63.64 \pm 1.86$ & $14.15 \pm 0.65 \mathrm{ab}$ \\
\hline & $\mathrm{F}$ & $36.73 \pm 1.78$ & $9.23 \pm 0.35$ & $66.52 \pm 4.55$ & $13.12 \pm 0.41 \mathrm{a}$ \\
\hline Sign & & ns & ns & ns & $*$ \\
\hline \multirow{3}{*}{ Cinsault } & $\mathrm{C}$ & $53.11 \pm 2.73$ & $14.81 \pm 0.71$ & $74.07 \pm 8.01$ & $18.68 \pm 2.11$ \\
\hline & $\mathrm{D}$ & $59.00 \pm 8.07$ & $16.98 \pm 1.38$ & $81.53 \pm 12.35$ & $18.41 \pm 1.98$ \\
\hline & $\mathrm{E}$ & $61.22 \pm 2.56$ & $16.47 \pm 1.04$ & $89.56 \pm 7.10$ & $16.14 \pm 0.51$ \\
\hline Sign & & ns & ns & ns & ns \\
\hline \multirow{3}{*}{ Croatina } & $\mathrm{D}$ & $41.23 \pm 1.47$ & $10.21 \pm 0.31$ & $69.05 \pm 4.09$ & $14.47 \pm 0.11$ \\
\hline & $\mathrm{E}$ & $43.99 \pm 2.10$ & $11.12 \pm 0.89$ & $73.70 \pm 1.46$ & $14.88 \pm 0.75$ \\
\hline & $\mathrm{F}$ & $45.04 \pm 4.53$ & $11.45 \pm 1.53$ & $75.17 \pm 4.87$ & $15.09 \pm 0.75$ \\
\hline Sign & & ns & ns & $\mathrm{ns}$ & $\mathrm{ns}$ \\
\hline \multirow{3}{*}{ Dolcetto } & $\mathrm{D}$ & $38.74 \pm 3.48$ & $9.65 \pm 1.48$ & $65.12 \pm 1.99$ & $14.64 \pm 1.17$ \\
\hline & $\mathrm{E}$ & $37.57 \pm 0.19$ & $9.08 \pm 0.55$ & $66.66 \pm 4.72$ & $14.35 \pm 0.86$ \\
\hline & $\mathrm{F}$ & $37.76 \pm 1.26$ & $8.93 \pm 0.44$ & $69.09 \pm 3.44$ & $13.28 \pm 0.29$ \\
\hline Sign & & $\mathrm{ns}$ & $\mathrm{ns}$ & $\mathrm{ns}$ & $\mathrm{ns}$ \\
\hline \multirow{3}{*}{ Erbaluce } & $\mathrm{D}$ & $35.49 \pm 0.37 a$ & $9.25 \pm 0.64 \mathrm{ab}$ & $64.50 \pm 2.87$ & $13.24 \pm 1.07$ \\
\hline & $\mathrm{E}$ & $39.29 \pm 0.58 b$ & $10.03 \pm 0.14 b$ & $72.08 \pm 2.48$ & $14.20 \pm 1.97$ \\
\hline & $\mathrm{F}$ & $35.38 \pm 2.18 \mathrm{a}$ & $8.72 \pm 0.30 \mathrm{a}$ & $70.03 \pm 5.62$ & $12.04 \pm 0.19$ \\
\hline Sign & & $*$ & $*$ & $\mathrm{~ns}$ & ns \\
\hline \multirow{3}{*}{ Freisa } & $\mathrm{D}$ & $50.60 \pm 4.93$ & $14.88 \pm 1.56$ & $75.44 \pm 9.21$ & $16.44 \pm 0.73$ \\
\hline & $\mathrm{E}$ & $56.20 \pm 4.25$ & $18.13 \pm 2.56$ & $74.47 \pm 3.21$ & $18.32 \pm 1.43$ \\
\hline & $\mathrm{F}$ & $56.33 \pm 1.26$ & $17.20 \pm 0.31$ & $79.67 \pm 3.82$ & $17.90 \pm 1.04$ \\
\hline Sign & & ns & $\mathrm{ns}$ & ns & $\mathrm{ns}$ \\
\hline \multirow{3}{*}{ Grenache } & $\mathrm{D}$ & $32.89 \pm 0.18$ & $8.42 \pm 0.03$ & $53.89 \pm 1.16$ & $14.28 \pm 0.08$ \\
\hline & $\mathrm{E}$ & $31.94 \pm 2.12$ & $8.18 \pm 1.06$ & $51.81 \pm 1.75$ & $16.29 \pm 1.81$ \\
\hline & $\mathrm{F}$ & $32.69 \pm 0.71$ & $8.46 \pm 0.34$ & $54.77 \pm 1.47$ & $14.84 \pm 0.67$ \\
\hline Sign & & ns & $\mathrm{ns}$ & $\mathrm{ns}$ & $\mathrm{ns}$ \\
\hline \multirow{3}{*}{ Grignolino } & $\mathrm{D}$ & $39.25 \pm 3.53$ & $10.50 \pm 0.77$ & $67.81 \pm 6.69$ & $13.57 \pm 0.43 a$ \\
\hline & $\mathrm{E}$ & $39.28 \pm 2.76$ & $10.66 \pm 1.07$ & $67.36 \pm 1.30$ & $14.52 \pm 0.27 b$ \\
\hline & $\mathrm{F}$ & $37.95 \pm 1.06$ & $9.62 \pm 0.61$ & $68.87 \pm 2.51$ & $13.66 \pm 0.26 \mathrm{a}$ \\
\hline Sign & & ns & $\mathrm{ns}$ & ns & $*$ \\
\hline \multirow{3}{*}{ Malvasia Bianca } & $\mathrm{D}$ & $47.88 \pm 4.91$ & $12.38 \pm 2.18$ & $77.84 \pm 1.08$ & $15.11 \pm 1.30$ \\
\hline & $\mathrm{E}$ & $45.76 \pm 1.88$ & $11.53 \pm 0.49$ & $74.86 \pm 6.28$ & $14.86 \pm 0.97$ \\
\hline & $\mathrm{F}$ & $46.48 \pm 3.04$ & $11.91 \pm 1.74$ & $78.34 \pm 0.19$ & $14.69 \pm 1.07$ \\
\hline Sign & & $\mathrm{ns}$ & $\mathrm{ns}$ & ns & $\mathrm{ns}$ \\
\hline \multirow{3}{*}{$\begin{array}{l}\text { Malvasia di } \\
\text { Schierano }\end{array}$} & $\mathrm{D}$ & $34.19 \pm 2.33$ & $8.90 \pm 0.60$ & $56.32 \pm 5.73$ & $14.80 \pm 0.87$ \\
\hline & $\mathrm{E}$ & $36.25 \pm 2.66$ & $9.53 \pm 1.00$ & $60.96 \pm 3.54$ & $14.55 \pm 0.26$ \\
\hline & $\mathrm{F}$ & $34.26 \pm 1.86$ & $8.96 \pm 0.87$ & $59.93 \pm 1.92$ & $13.99 \pm 0.60$ \\
\hline Sign & & ns & $\mathrm{ns}$ & $\mathrm{ns}$ & $\mathrm{ns}$ \\
\hline \multirow{3}{*}{ Merlot } & $\mathrm{D}$ & $47.31 \pm 2.65$ & $13.03 \pm 1.29$ & $77.46 \pm 1.97 \mathrm{a}$ & $14.55 \pm 0.90 b$ \\
\hline & $\mathrm{E}$ & $46.94 \pm 3.71$ & $11.43 \pm 1.22$ & $86.82 \pm 1.64 b$ & $12.99 \pm 0.57 \mathrm{a}$ \\
\hline & $\mathrm{F}$ & $47.04 \pm 1.50$ & $11.43 \pm 0.85$ & $90.43 \pm 2.51 b$ & $12.71 \pm 0.31 \mathrm{a}$ \\
\hline Sign & & ns & $\mathrm{ns}$ & $* * *$ & $*$ \\
\hline \multirow{3}{*}{ Moscato Bianco } & $\mathrm{D}$ & $31.91 \pm 1.76 \mathrm{a}$ & $8.01 \pm 0.49 a$ & $57.19 \pm 2.67 \mathrm{a}$ & $13.49 \pm 0.11 \mathrm{a}$ \\
\hline & $\mathrm{E}$ & $36.76 \pm 0.21 b$ & $9.60 \pm 0.47 b$ & $64.82 \pm 1.15 b$ & $14.21 \pm 0.14 b$ \\
\hline & $\mathrm{F}$ & $35.21 \pm 1.50 \mathrm{~b}$ & $8.84 \pm 0.46 \mathrm{ab}$ & $61.77 \pm 4.02 \mathrm{ab}$ & $14.04 \pm 0.21 b$ \\
\hline Sign & & $*$ & $*$ & $*$ & $* *$ \\
\hline
\end{tabular}


TABLE 2 (CONTINUED)

\begin{tabular}{|c|c|c|c|c|c|}
\hline Grape variety & Density class & $\mathrm{F}_{\mathrm{s}}(\mathrm{N})$ & $\mathrm{W}_{\mathrm{s}}(\mathrm{mJ})$ & $\mathrm{E}_{\mathrm{s}}(\mathrm{N} / \mathrm{mm})$ & $\mathrm{DI}_{\mathrm{s}}(\%)$ \\
\hline \multirow{3}{*}{ Mourvèdre } & $\mathrm{D}$ & $52.06 \pm 3.31$ & $14.73 \pm 1.83$ & $79.89 \pm 2.44$ & $14.74 \pm 0.46$ \\
\hline & $\mathrm{E}$ & $49.76 \pm 1.11$ & $14.25 \pm 1.38$ & $75.65 \pm 3.84$ & $14.73 \pm 0.97$ \\
\hline & $\mathrm{F}$ & $48.71 \pm 1.60$ & $13.60 \pm 1.10$ & $77.81 \pm 2.22$ & $13.56 \pm 0.24$ \\
\hline \multirow[t]{2}{*}{ Sign } & & $\mathrm{ns}$ & $\mathrm{ns}$ & $\mathrm{ns}$ & $\mathrm{ns}$ \\
\hline & $\mathrm{D}$ & $53.29 \pm 4.06$ & $14.58 \pm 1.94$ & $81.45 \pm 1.37$ & $16.80 \pm 1.36$ \\
\hline \multirow[t]{2}{*}{ Nebbiolo } & $\mathrm{E}$ & $47.30 \pm 0.82$ & $12.39 \pm 0.82$ & $78.86 \pm 1.57$ & $15.24 \pm 0.26$ \\
\hline & $\mathrm{F}$ & $45.46 \pm 3.73$ & $11.09 \pm 1.40$ & $80.16 \pm 2.62$ & $14.94 \pm 0.99$ \\
\hline \multirow[t]{2}{*}{ Sign } & & ns & ns & ns & ns \\
\hline & $\mathrm{D}$ & $55.32 \pm 0.62 b$ & $15.84 \pm 0.15 b$ & $83.74 \pm 2.84$ & $14.93 \pm 0.39$ \\
\hline \multirow[t]{2}{*}{ Petit Rouge } & $\mathrm{E}$ & $49.76 \pm 1.45 \mathrm{a}$ & $12.70 \pm 0.88 \mathrm{a}$ & $86.87 \pm 2.03$ & $13.50 \pm 0.71$ \\
\hline & $\mathrm{F}$ & $48.78 \pm 2.64 a$ & $12.67 \pm 0.68 \mathrm{a}$ & $81.53 \pm 6.67$ & $14.26 \pm 0.65$ \\
\hline \multirow[t]{2}{*}{ Sign } & & $* *$ & $* *$ & $\mathrm{~ns}$ & $\mathrm{~ns}$ \\
\hline & $\mathrm{D}$ & $52.74 \pm 0.64 a$ & $14.96 \pm 0.77$ & $80.82 \pm 3.76$ & $16.41 \pm 0.98$ \\
\hline \multirow[t]{2}{*}{ Petit Verdot } & $\mathrm{E}$ & $52.60 \pm 0.47 \mathrm{a}$ & $14.24 \pm 0.38$ & $83.52 \pm 3.31$ & $16.19 \pm 0.44$ \\
\hline & $\mathrm{F}$ & $55.45 \pm 0.19 b$ & $15.01 \pm 0.54$ & $89.44 \pm 4.17$ & $16.42 \pm 0.57$ \\
\hline \multirow[t]{2}{*}{ Sign } & & $* * *$ & ns & ns & $\mathrm{ns}$ \\
\hline & $\mathrm{D}$ & $47.23 \pm 1.93$ & $12.78 \pm 0.26$ & $77.39 \pm 3.70$ & $15.53 \pm 0.48$ \\
\hline \multirow[t]{2}{*}{ Pignolo Spano } & $\mathrm{E}$ & $48.35 \pm 1.66$ & $13.24 \pm 0.21$ & $81.92 \pm 6.13$ & $16.52 \pm 1.87$ \\
\hline & $\mathrm{F}$ & $50.81 \pm 2.17$ & $13.39 \pm 1.15$ & $84.98 \pm 1.16$ & $15.68 \pm 0.50$ \\
\hline \multirow[t]{2}{*}{ Sign } & & ns & ns & ns & ns \\
\hline & $\mathrm{D}$ & $38.46 \pm 2.36$ & $12.37 \pm 1.39$ & $51.73 \pm 2.24 a$ & $18.88 \pm 1.06 b$ \\
\hline \multirow[t]{2}{*}{ Pinot Noir } & $\mathrm{E}$ & $41.98 \pm 1.75$ & $12.27 \pm 1.27$ & $62.73 \pm 1.60 b$ & $15.49 \pm 0.89 a$ \\
\hline & $\mathrm{F}$ & $41.96 \pm 3.61$ & $11.96 \pm 1.99$ & $66.72 \pm 1.20 \mathrm{c}$ & $15.15 \pm 1.38 \mathrm{a}$ \\
\hline \multirow[t]{2}{*}{ Sign } & & $\mathrm{ns}$ & $\mathrm{ns}$ & $* * *$ & $*$ \\
\hline & $\mathrm{D}$ & $34.87 \pm 1.21$ & $9.35 \pm 0.87$ & $59.88 \pm 1.71 \mathrm{a}$ & $14.81 \pm 0.54$ \\
\hline \multirow[t]{2}{*}{ Riesling Italico } & $\mathrm{E}$ & $37.96 \pm 2.79$ & $9.99 \pm 1.14$ & $65.82 \pm 2.13 b$ & $16.23 \pm 2.44$ \\
\hline & $\mathrm{F}$ & $37.85 \pm 0.96$ & $9.93 \pm 0.37$ & $65.54 \pm 1.52 b$ & $16.25 \pm 2.50$ \\
\hline \multirow[t]{2}{*}{ Sign } & & ns & ns & $*$ & ns \\
\hline & $\mathrm{D}$ & $38.80 \pm 1.87$ & $10.52 \pm 0.39$ & $65.91 \pm 5.06$ & $14.38 \pm 0.47$ \\
\hline \multirow[t]{2}{*}{ Ruchè } & $\mathrm{E}$ & $41.36 \pm 0.34$ & $11.29 \pm 0.48$ & $71.20 \pm 1.71$ & $14.63 \pm 0.43$ \\
\hline & $\mathrm{F}$ & $40.05 \pm 2.21$ & $10.25 \pm 0.90$ & $72.88 \pm 1.72$ & $13.67 \pm 0.08$ \\
\hline \multirow[t]{2}{*}{ Sign } & & ns & ns & ns & ns \\
\hline & $\mathrm{C}$ & $51.85 \pm 2.02$ & $15.40 \pm 1.00$ & $72.90 \pm 2.56$ & $16.39 \pm 0.56$ \\
\hline \multirow[t]{2}{*}{ Sangiovese } & $\mathrm{D}$ & $53.02 \pm 2.30$ & $16.31 \pm 0.80$ & $69.11 \pm 3.53$ & $17.35 \pm 0.30$ \\
\hline & $\mathrm{E}$ & $50.86 \pm 0.75$ & $15.16 \pm 0.21$ & $69.68 \pm 5.22$ & $16.75 \pm 0.56$ \\
\hline \multirow[t]{2}{*}{ Sign } & & ns & $\mathrm{ns}$ & $\mathrm{ns}$ & $\mathrm{ns}$ \\
\hline & $\mathrm{D}$ & $38.12 \pm 7.82 \mathrm{a}$ & $10.18 \pm 2.20$ & $64.66 \pm 12.13 a$ & $13.88 \pm 1.33$ \\
\hline \multirow[t]{2}{*}{ Sauvignon Blanc } & $\mathrm{E}$ & $48.59 \pm 1.87 b$ & $12.40 \pm 0.57$ & $83.51 \pm 2.03 b$ & $13.78 \pm 0.39$ \\
\hline & $\mathrm{F}$ & $48.27 \pm 0.40 b$ & $12.23 \pm 0.74$ & $86.75 \pm 4.74 b$ & $13.56 \pm 0.77$ \\
\hline \multirow[t]{2}{*}{ Sign } & & $*$ & $\mathrm{~ns}$ & $*$ & $\mathrm{~ns}$ \\
\hline & $\mathrm{D}$ & $35.63 \pm 1.48$ & $9.45 \pm 0.64$ & $58.86 \pm 1.86$ & $14.90 \pm 0.37$ \\
\hline \multirow[t]{2}{*}{ Syrah } & $\mathrm{E}$ & $35.87 \pm 1.37$ & $9.53 \pm 0.87$ & $59.72 \pm 0.98$ & $14.85 \pm 0.41$ \\
\hline & $\mathrm{F}$ & $34.20 \pm 4.17$ & $9.54 \pm 2.04$ & $54.97 \pm 5.25$ & $15.75 \pm 2.16$ \\
\hline Sign & & ns & $\mathrm{ns}$ & ns & ns \\
\hline
\end{tabular}

Data are expressed as mean value \pm standard deviation $(n=3)$. Different Latin letters within the same column indicate significant differences $\left(^{\mathrm{a}}\right)$ among density classes according to the Tukey-b test $(p<0.05)$. $\operatorname{Sign}^{\mathrm{a}}$ : *,**,*** indicate significance at $p<0.05$, 0.01 and 0.001 , respectively. $\mathrm{ns}=$ not significant. $\mathrm{F}_{\mathrm{s}}=$ seed break force, $\mathrm{W}_{\mathrm{s}}=$ seed break energy, $\mathrm{E}_{\mathrm{s}}=$ resistance of the seed to axial deformation, $\mathrm{DI}_{\mathrm{s}}=$ seed deformation index. $\mathrm{C}=1088 \mathrm{~kg} / \mathrm{m}^{3}, \mathrm{D}=1094 \mathrm{~kg} / \mathrm{m}^{3}, \mathrm{E}=1100 \mathrm{~kg} / \mathrm{m}^{3}, \mathrm{~F}=1107 \mathrm{~kg} / \mathrm{m}^{3}$. 
factors ranging from 0.486 to 0.943 ( $p<0.01$ ). A work recently published also highlighted that the seed texture properties measured by compression testing are positively correlated with each other (Letaief et al., 2013).

The differentiating power of $\mathrm{F}_{\mathrm{s}}$ is shown in Fig. 2, where the wine grape varieties studied were classified according to this compression parameter by cluster analysis. The varieties characterised by the softest seeds $\left(\mathrm{F}_{\mathrm{s}}\right.$ values ranging from 32.51 to $40.80 \mathrm{~N}$ ) were included in the first cluster (upper side), whereas those having the hardest seeds ( $F_{s}$ values between 42.84 and $57.78 \mathrm{~N}$ ) were grouped in the second cluster (bottom side). Nevertheless, the Cinsault cultivar was well differentiated inside this last cluster, and the other subcluster was composed of two other groups, well separated, including those varieties with $\mathrm{F}_{\mathrm{s}}$ values ranging from 42.84 to $44.99 \mathrm{~N}$ and from 46.71 to $54.38 \mathrm{~N}$. The differences found in seed hardness among the wine grape varieties studied showed that this mechanical parameter can be considered an ampelographic characteristic of each variety independently of the berry ripening grade, and therefore an efficient varietal marker.
Prediction of the extractable content of phenolic compounds from seed mechanical properties

Table 3 shows the reference values of some spectrophotometric indices often used in wineries to evaluate the phenolic composition extractable from the seeds into the wine-like solution for all wine grape varieties studied at the three ripening stages defined by berry density. Thus, the high natural variability in the quantitative phenolic composition of the seeds was considered. A total of 90 seed samples were analysed. Table 4 summarises the reference values for the extractable content of gallic acid and monomeric and dimeric flavanols in the same samples of seeds. All results were expressed per grape weight. For each wine grape variety, the spectrophotometric indices of the seeds were similar for berries belonging to different density classes, with very few exceptions. The lowest values of absorbance at $280 \mathrm{~nm}$ $\left(\mathrm{A}_{280}\right)$, as well as of the extractable content of total flavonoids (TF) and flavanols reactive to vanillin (FRV), corresponded to the Cinsault cultivar, followed by Grenache, whereas those of the extractable content of proanthocyanidins (PRO) were associated with the Cinsault cultivar, followed by Barbera and Sauvignon Blanc. In contrast, Petit Verdot and Pinot
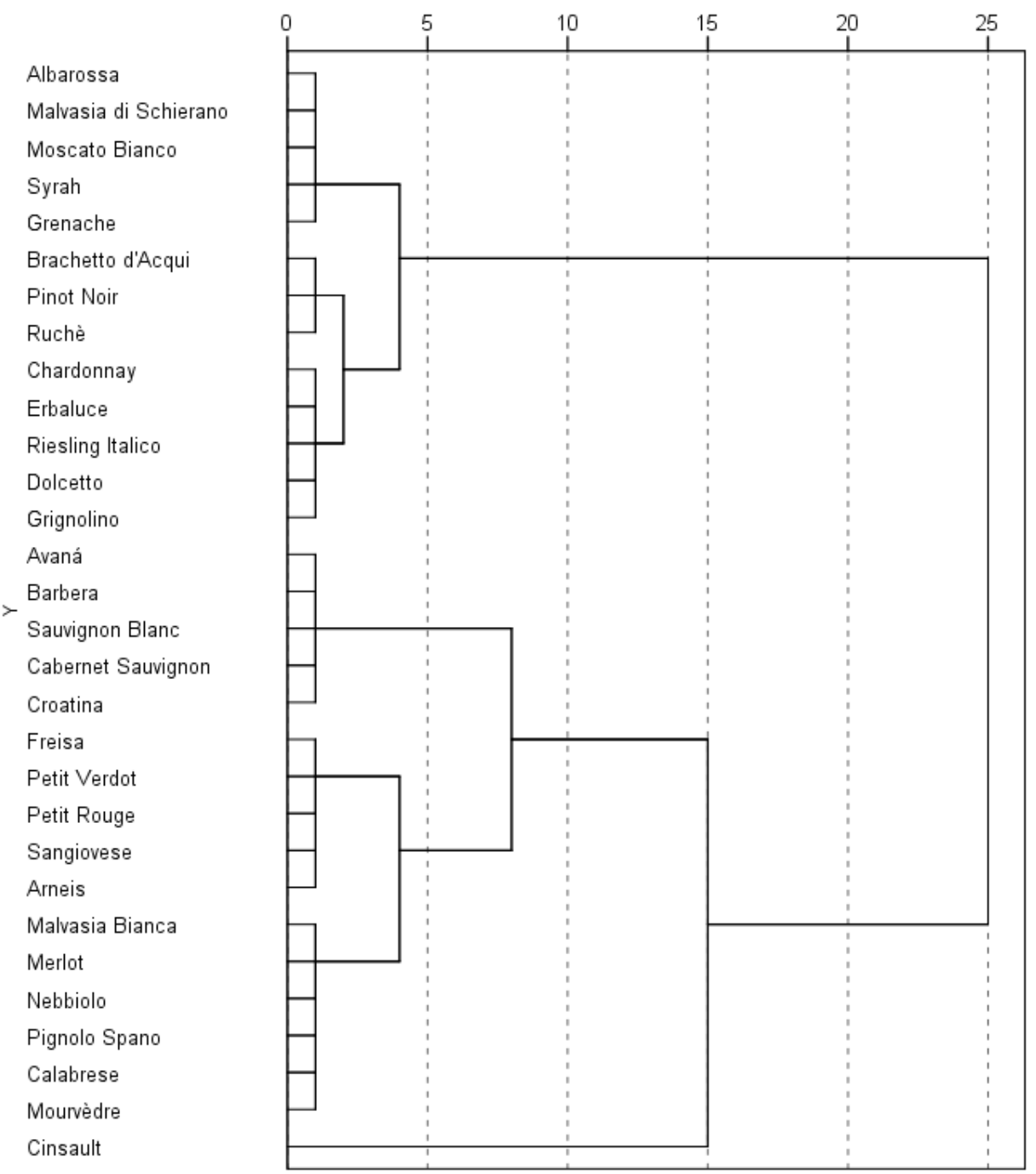

FIGURE 2

Dendogram of wine grape varieties by applying the average linkage between groups and squared Euclidean distance for hierarchical cluster analysis according to the seed break force. 
TABLE 3

Spectrophotometric indices related to the phenolic composition of seeds per grape weight from densimetrically sorted berries at harvest.

\begin{tabular}{|c|c|c|c|c|c|c|}
\hline Grape variety & Density class & $\mathrm{A}_{280}(1 / \mathrm{kg})$ & $\mathrm{TF}(\mathrm{mg} / \mathrm{kg})$ & PRO $(\mathrm{mg} / \mathrm{kg})$ & $\mathrm{FRV}(\mathrm{mg} / \mathrm{kg})$ & FRV/PRO \\
\hline \multirow{3}{*}{ Albarossa } & $\mathrm{D}$ & $43.5 \pm 4.0$ & $3606 \pm 261$ & $2391 \pm 226 b$ & $1677 \pm 185$ & $0.70 \pm 0.05$ \\
\hline & $\mathrm{E}$ & $36.5 \pm 2.9$ & $3136 \pm 238$ & $1851 \pm 119 a$ & $1441 \pm 144$ & $0.78 \pm 0.06$ \\
\hline & $\mathrm{F}$ & $43.0 \pm 5.8$ & $3581 \pm 411$ & $2309 \pm 237 b$ & $1779 \pm 384$ & $0.76 \pm 0.08$ \\
\hline $\operatorname{Sign}^{\mathrm{a}}$ & & ns & ns & $*$ & ns & ns \\
\hline \multirow{3}{*}{ Arneis } & $\mathrm{D}$ & $38.4 \pm 1.1$ & $2945 \pm 33$ & $1945 \pm 40$ & $1619 \pm 144$ & $0.83 \pm 0.06$ \\
\hline & $\mathrm{E}$ & $37.6 \pm 5.4$ & $2918 \pm 298$ & $2026 \pm 230$ & $1674 \pm 160$ & $0.83 \pm 0.03$ \\
\hline & $\mathrm{F}$ & $35.3 \pm 6.5$ & $2684 \pm 469$ & $1787 \pm 307$ & $1484 \pm 214$ & $0.83 \pm 0.03$ \\
\hline Sign & & ns & ns & ns & ns & ns \\
\hline \multirow{3}{*}{ Avaná } & $\mathrm{D}$ & $28.3 \pm 2.0$ & $2026 \pm 171$ & $1271 \pm 208$ & $1065 \pm 92$ & $0.85 \pm 0.08$ \\
\hline & $\mathrm{E}$ & $26.2 \pm 2.5$ & $1986 \pm 199$ & $1238 \pm 143$ & $972 \pm 107$ & $0.79 \pm 0.09$ \\
\hline & $\mathrm{F}$ & $23.9 \pm 3.5$ & $1752 \pm 247$ & $1088 \pm 137$ & $951 \pm 187$ & $0.87 \pm 0.07$ \\
\hline Sign & & ns & $\mathrm{ns}$ & $\mathrm{ns}$ & ns & $\mathrm{ns}$ \\
\hline \multirow{3}{*}{ Barbera } & $\mathrm{D}$ & $30.0 \pm 5.5$ & $2152 \pm 372$ & $1090 \pm 215$ & $1063 \pm 87$ & $0.99 \pm 0.13$ \\
\hline & $\mathrm{E}$ & $26.3 \pm 2.1$ & $1975 \pm 110$ & $1040 \pm 105$ & $1025 \pm 79$ & $0.99 \pm 0.03$ \\
\hline & $\mathrm{F}$ & $24.7 \pm 2.6$ & $2009 \pm 194$ & $1114 \pm 242$ & $984 \pm 106$ & $0.90 \pm 0.18$ \\
\hline Sign & & ns & ns & ns & ns & ns \\
\hline \multirow{3}{*}{ Brachetto d'Acqui } & $\mathrm{D}$ & $60.9 \pm 11.6$ & $4328 \pm 576$ & $2573 \pm 321$ & $2124 \pm 254$ & $0.83 \pm 0.02$ \\
\hline & $\mathrm{E}$ & $48.1 \pm 2.4$ & $3421 \pm 244$ & $2038 \pm 226$ & $1696 \pm 159$ & $0.83 \pm 0.02$ \\
\hline & $\mathrm{F}$ & $53.2 \pm 8.3$ & $3620 \pm 389$ & $2236 \pm 381$ & $1896 \pm 125$ & $0.86 \pm 0.11$ \\
\hline Sign & & $\mathrm{ns}$ & $\mathrm{ns}$ & ns & $\mathrm{ns}$ & $\mathrm{ns}$ \\
\hline \multirow{3}{*}{$\begin{array}{l}\text { Cabernet } \\
\text { Sauvignon }\end{array}$} & $\mathrm{D}$ & $45.7 \pm 8.5$ & $3426 \pm 463$ & $2603 \pm 142$ & $1904 \pm 45$ & $0.73 \pm 0.03$ \\
\hline & $\mathrm{E}$ & $48.4 \pm 11.0$ & $3790 \pm 667$ & $2887 \pm 397$ & $2006 \pm 158$ & $0.70 \pm 0.04$ \\
\hline & $\mathrm{F}$ & $46.9 \pm 4.1$ & $3684 \pm 291$ & $2620 \pm 591$ & $1894 \pm 187$ & $0.74 \pm 0.11$ \\
\hline Sign & & ns & ns & ns & ns & ns \\
\hline \multirow{3}{*}{ Calabrese } & $\mathrm{D}$ & $36.5 \pm 1.2$ & $2798 \pm 229$ & $1477 \pm 22$ & $1585 \pm 70$ & $1.07 \pm 0.06 \mathrm{ab}$ \\
\hline & $\mathrm{E}$ & $33.3 \pm 3.9$ & $2394 \pm 324$ & $1401 \pm 246$ & $1387 \pm 140$ & $1.00 \pm 0.08 \mathrm{a}$ \\
\hline & $\mathrm{F}$ & $31.9 \pm 0.9$ & $2273 \pm 115$ & $1183 \pm 55$ & $1413 \pm 62$ & $1.19 \pm 0.02 b$ \\
\hline Sign & & ns & ns & ns & ns & $*$ \\
\hline \multirow{3}{*}{ Chardonnay } & $\mathrm{D}$ & $40.4 \pm 7.0$ & $2951 \pm 347$ & $1879 \pm 364$ & $1777 \pm 263$ & $0.95 \pm 0.05$ \\
\hline & $\mathrm{E}$ & $37.2 \pm 2.7$ & $2948 \pm 285$ & $1764 \pm 214$ & $1725 \pm 152$ & $0.98 \pm 0.03$ \\
\hline & $\mathrm{F}$ & $32.0 \pm 1.6$ & $2516 \pm 33$ & $1583 \pm 50$ & $1489 \pm 32$ & $0.94 \pm 0.04$ \\
\hline Sign & & $\mathrm{ns}$ & $\mathrm{ns}$ & $\mathrm{ns}$ & $\mathrm{ns}$ & $\mathrm{ns}$ \\
\hline \multirow{3}{*}{ Cinsault } & $\mathrm{C}$ & $19.1 \pm 2.7$ & $1521 \pm 222$ & $984 \pm 124$ & $802 \pm 118$ & $0.81 \pm 0.05$ \\
\hline & $\mathrm{D}$ & $16.6 \pm 2.8$ & $1301 \pm 197$ & $832 \pm 161$ & $625 \pm 140$ & $0.75 \pm 0.08$ \\
\hline & $\mathrm{E}$ & $16.2 \pm 1.3$ & $1266 \pm 51$ & $829 \pm 95$ & $654 \pm 77$ & $0.79 \pm 0.11$ \\
\hline Sign & & $\mathrm{ns}$ & ns & ns & ns & $\mathrm{ns}$ \\
\hline \multirow{3}{*}{ Croatina } & $\mathrm{D}$ & $45.2 \pm 6.0$ & $3370 \pm 330$ & $1950 \pm 266$ & $1792 \pm 251$ & $0.92 \pm 0.04$ \\
\hline & $\mathrm{E}$ & $42.7 \pm 2.3$ & $3169 \pm 134$ & $1914 \pm 25$ & $1606 \pm 67$ & $0.84 \pm 0.04$ \\
\hline & $\mathrm{F}$ & $42.6 \pm 3.5$ & $3175 \pm 228$ & $1786 \pm 203$ & $1693 \pm 134$ & $0.95 \pm 0.10$ \\
\hline Sign & & ns & ns & ns & ns & ns \\
\hline \multirow{3}{*}{ Dolcetto } & $\mathrm{D}$ & $36.8 \pm 6.8$ & $2803 \pm 273$ & $1879 \pm 359$ & $1351 \pm 112$ & $0.73 \pm 0.09$ \\
\hline & $\mathrm{E}$ & $34.3 \pm 5.7$ & $2620 \pm 504$ & $1683 \pm 383$ & $1242 \pm 218$ & $0.75 \pm 0.06$ \\
\hline & $\mathrm{F}$ & $38.4 \pm 5.7$ & $2895 \pm 428$ & $1881 \pm 181$ & $1467 \pm 275$ & $0.78 \pm 0.07$ \\
\hline Sign & & $\mathrm{ns}$ & $\mathrm{ns}$ & ns & $\mathrm{ns}$ & $\mathrm{ns}$ \\
\hline
\end{tabular}


TABLE 3 (CONTINUED)

\begin{tabular}{|c|c|c|c|c|c|c|}
\hline Grape variety & Density class & $\mathrm{A}_{280}(1 / \mathrm{kg})$ & $\mathrm{TF}(\mathrm{mg} / \mathrm{kg})$ & PRO (mg/kg) & FRV $(\mathrm{mg} / \mathrm{kg})$ & FRV/PRO \\
\hline \multirow{3}{*}{ Erbaluce } & $\mathrm{D}$ & $25.4 \pm 2.6$ & $2057 \pm 80$ & $1360 \pm 109$ & $1026 \pm 37$ & $0.76 \pm 0.06$ \\
\hline & $\mathrm{E}$ & $28.4 \pm 1.1$ & $2101 \pm 147$ & $1394 \pm 34$ & $1041 \pm 46$ & $0.75 \pm 0.02$ \\
\hline & $\mathrm{F}$ & $25.5 \pm 3.1$ & $1891 \pm 73$ & $1250 \pm 57$ & $906 \pm 88$ & $0.73 \pm 0.09$ \\
\hline Sign & & ns & ns & ns & ns & ns \\
\hline \multirow{3}{*}{ Freisa } & D & $55.7 \pm 4.3$ & $4031 \pm 373$ & $2325 \pm 262$ & $1570 \pm 108$ & $0.68 \pm 0.03$ \\
\hline & E & $55.9 \pm 4.6$ & $3803 \pm 511$ & $2295 \pm 175$ & $1446 \pm 227$ & $0.63 \pm 0.05$ \\
\hline & $\mathrm{F}$ & $48.9 \pm 5.2$ & $3467 \pm 116$ & $2041 \pm 50$ & $1323 \pm 93$ & $0.65 \pm 0.03$ \\
\hline Sign & & ns & ns & ns & ns & ns \\
\hline \multirow{3}{*}{ Grenache } & $\mathrm{D}$ & $23.7 \pm 1.7$ & $1862 \pm 133$ & $1303 \pm 109$ & $842 \pm 59$ & $0.65 \pm 0.02$ \\
\hline & E & $22.5 \pm 6.0$ & $1776 \pm 497$ & $1297 \pm 307$ & $814 \pm 221$ & $0.62 \pm 0.03$ \\
\hline & $\mathrm{F}$ & $23.9 \pm 0.8$ & $1868 \pm 68$ & $1351 \pm 54$ & $888 \pm 85$ & $0.66 \pm 0.06$ \\
\hline Sign & & ns & ns & ns & ns & ns \\
\hline \multirow{3}{*}{ Grignolino } & D & $37.3 \pm 8.1$ & $3149 \pm 404$ & $2346 \pm 470$ & $1817 \pm 219$ & $0.78 \pm 0.08$ \\
\hline & E & $42.6 \pm 3.2$ & $3315 \pm 93$ & $2510 \pm 215$ & $1859 \pm 136$ & $0.74 \pm 0.02$ \\
\hline & $\mathrm{F}$ & $33.6 \pm 2.2$ & $3008 \pm 333$ & $2407 \pm 443$ & $1691 \pm 157$ & $0.71 \pm 0.08$ \\
\hline Sign & & ns & ns & ns & ns & ns \\
\hline \multirow{3}{*}{ Malvasia Bianca } & $\mathrm{D}$ & $30.6 \pm 0.8$ & $2181 \pm 328$ & $1419 \pm 200$ & $1063 \pm 174$ & $0.75 \pm 0.04$ \\
\hline & $\mathrm{E}$ & $36.2 \pm 6.0$ & $2624 \pm 358$ & $1664 \pm 328$ & $1261 \pm 201$ & $0.76 \pm 0.07$ \\
\hline & $\mathrm{F}$ & $29.2 \pm 4.9$ & $2178 \pm 280$ & $1480 \pm 285$ & $1209 \pm 223$ & $0.82 \pm 0.07$ \\
\hline Sign & & ns & ns & ns & ns & ns \\
\hline \multirow{3}{*}{$\begin{array}{l}\text { Malvasia di } \\
\text { Schierano }\end{array}$} & $\mathrm{D}$ & $33.6 \pm 3.1$ & $2465 \pm 122$ & $1454 \pm 48$ & $1204 \pm 87$ & $0.83 \pm 0.04$ \\
\hline & E & $37.1 \pm 6.1$ & $2746 \pm 315$ & $1686 \pm 166$ & $1341 \pm 167$ & $0.79 \pm 0.05$ \\
\hline & $\mathrm{F}$ & $37.6 \pm 5.2$ & $2810 \pm 350$ & $1745 \pm 270$ & $1437 \pm 109$ & $0.83 \pm 0.09$ \\
\hline Sign & & ns & ns & ns & ns & ns \\
\hline \multirow{3}{*}{ Merlot } & $\mathrm{D}$ & $40.0 \pm 4.6$ & $2971 \pm 119$ & $2095 \pm 162$ & $1799 \pm 92$ & $0.86 \pm 0.07$ \\
\hline & E & $40.7 \pm 3.1$ & $2979 \pm 36$ & $2094 \pm 113$ & $1741 \pm 15$ & $0.83 \pm 0.04$ \\
\hline & $\mathrm{F}$ & $39.0 \pm 2.0$ & $2830 \pm 471$ & $2250 \pm 64$ & $1771 \pm 70$ & $0.79 \pm 0.03$ \\
\hline Sign & & $\mathrm{ns}$ & $\mathrm{ns}$ & $\mathrm{ns}$ & $\mathrm{ns}$ & $\mathrm{ns}$ \\
\hline \multirow{3}{*}{ Moscato Bianco } & $\mathrm{D}$ & $36.2 \pm 4.8$ & $2652 \pm 306$ & $1653 \pm 179$ & $1260 \pm 43$ & $0.77 \pm 0.06$ \\
\hline & $\mathrm{E}$ & $37.3 \pm 2.1$ & $2590 \pm 114$ & $1487 \pm 84$ & $1297 \pm 73$ & $0.87 \pm 0.08$ \\
\hline & $\mathrm{F}$ & $34.8 \pm 2.4$ & $2541 \pm 87$ & $1541 \pm 135$ & $1255 \pm 74$ & $0.82 \pm 0.07$ \\
\hline Sign & & ns & $\mathrm{ns}$ & $\mathrm{ns}$ & ns & $\mathrm{ns}$ \\
\hline \multirow{3}{*}{ Mourvèdre } & D & $53.7 \pm 3.9$ & $4174 \pm 365$ & $2965 \pm 319$ & $1949 \pm 196$ & $0.66 \pm 0.04$ \\
\hline & E & $54.2 \pm 3.9$ & $4205 \pm 294$ & $3029 \pm 216$ & $1973 \pm 138$ & $0.65 \pm 0.06$ \\
\hline & $\mathrm{F}$ & $51.4 \pm 2.9$ & $3916 \pm 254$ & $2993 \pm 225$ & $2070 \pm 100$ & $0.70 \pm 0.08$ \\
\hline Sign & & $\mathrm{ns}$ & $\mathrm{ns}$ & $\mathrm{ns}$ & ns & ns \\
\hline \multirow{3}{*}{ Nebbiolo } & D & $35.1 \pm 4.2$ & $2613 \pm 515$ & $1702 \pm 186$ & $1314 \pm 132$ & $0.77 \pm 0.03$ \\
\hline & E & $30.0 \pm 4.9$ & $2453 \pm 313$ & $1551 \pm 161$ & $1193 \pm 53$ & $0.77 \pm 0.05$ \\
\hline & $\mathrm{F}$ & $30.5 \pm 1.8$ & $2377 \pm 183$ & $1661 \pm 114$ & $1198 \pm 59$ & $0.72 \pm 0.05$ \\
\hline Sign & & ns & $\mathrm{ns}$ & $\mathrm{ns}$ & $\mathrm{ns}$ & ns \\
\hline \multirow{3}{*}{ Petit Rouge } & $\mathrm{D}$ & $40.2 \pm 8.5$ & $2908 \pm 469$ & $2083 \pm 256$ & $1825 \pm 318$ & $0.87 \pm 0.06$ \\
\hline & E & $39.7 \pm 2.1$ & $2959 \pm 137$ & $1999 \pm 64$ & $1846 \pm 135$ & $0.92 \pm 0.05$ \\
\hline & $\mathrm{F}$ & $36.6 \pm 3.5$ & $2772 \pm 288$ & $1974 \pm 182$ & $1817 \pm 198$ & $0.92 \pm 0.02$ \\
\hline Sign & & ns & $\mathrm{ns}$ & ns & $\mathrm{ns}$ & ns \\
\hline \multirow{3}{*}{ Petit Verdot } & D & $65.9 \pm 1.5$ & $4676 \pm 144$ & $3043 \pm 61$ & $2546 \pm 127$ & $0.84 \pm 0.03 b$ \\
\hline & E & $66.3 \pm 7.7$ & $4728 \pm 424$ & $3281 \pm 387$ & $2422 \pm 320$ & $0.74 \pm 0.01 \mathrm{a}$ \\
\hline & $\mathrm{F}$ & $65.9 \pm 7.6$ & $4872 \pm 609$ & $3201 \pm 388$ & $2640 \pm 401$ & $0.82 \pm 0.06 \mathrm{~b}$ \\
\hline Sign & & ns & ns & ns & ns & * \\
\hline
\end{tabular}


TABLE 3 (CONTINUED)

\begin{tabular}{|c|c|c|c|c|c|c|}
\hline Grape variety & Density class & $\mathrm{A}_{280}(1 / \mathrm{kg})$ & $\mathrm{TF}(\mathrm{mg} / \mathrm{kg})$ & PRO (mg/kg) & FRV (mg/kg) & FRV/PRO \\
\hline \multirow{3}{*}{ Pignolo Spano } & $\mathrm{D}$ & $42.5 \pm 1.1$ & $3403 \pm 78$ & $2176 \pm 36$ & $1791 \pm 6$ & $0.82 \pm 0.01$ \\
\hline & $\mathrm{E}$ & $42.8 \pm 0.8$ & $3435 \pm 49$ & $2245 \pm 74$ & $1843 \pm 61$ & $0.82 \pm 0.01$ \\
\hline & $\mathrm{F}$ & $43.5 \pm 1.4$ & $3463 \pm 124$ & $2297 \pm 90$ & $2068 \pm 334$ & $0.90 \pm 0.17$ \\
\hline \multirow[t]{2}{*}{ Sign } & & ns & $\mathrm{ns}$ & ns & $\mathrm{ns}$ & ns \\
\hline & $\mathrm{D}$ & $66.2 \pm 4.1$ & $4646 \pm 285$ & $2913 \pm 217$ & $3015 \pm 208$ & $1.04 \pm 0.01$ \\
\hline \multirow[t]{2}{*}{ Pinot Noir } & $\mathrm{E}$ & $61.9 \pm 6.2$ & $4661 \pm 472$ & $2926 \pm 91$ & $2850 \pm 288$ & $0.98 \pm 0.11$ \\
\hline & $\mathrm{F}$ & $65.3 \pm 10.2$ & $4684 \pm 147$ & $3039 \pm 343$ & $3036 \pm 223$ & $1.00 \pm 0.04$ \\
\hline \multirow[t]{2}{*}{ Sign } & & $\mathrm{ns}$ & $\mathrm{ns}$ & ns & ns & ns \\
\hline & $\mathrm{D}$ & $48.6 \pm 4.0$ & $3906 \pm 482$ & $2927 \pm 310$ & $2167 \pm 298$ & $0.74 \pm 0.04$ \\
\hline \multirow[t]{2}{*}{ Riesling Italico } & $\mathrm{E}$ & $44.3 \pm 4.9$ & $3569 \pm 318$ & $2647 \pm 166$ & $1974 \pm 187$ & $0.74 \pm 0.03$ \\
\hline & $\mathrm{F}$ & $45.3 \pm 6.2$ & $3632 \pm 474$ & $2661 \pm 520$ & $2158 \pm 233$ & $0.82 \pm 0.09$ \\
\hline \multirow[t]{2}{*}{ Sign } & & $\mathrm{ns}$ & $\mathrm{ns}$ & ns & ns & ns \\
\hline & $\mathrm{D}$ & $40.9 \pm 1.5 b$ & $3305 \pm 71$ & $2100 \pm 79$ & $1683 \pm 100$ & $0.80 \pm 0.02$ \\
\hline \multirow[t]{2}{*}{ Ruchè } & $\mathrm{E}$ & $38.1 \pm 0.8 \mathrm{ab}$ & $2948 \pm 221$ & $1889 \pm 226$ & $1611 \pm 96$ & $0.86 \pm 0.08$ \\
\hline & $\mathrm{F}$ & $36.1 \pm 2.2 \mathrm{a}$ & $2917 \pm 242$ & $1917 \pm 148$ & $1793 \pm 269$ & $0.93 \pm 0.10$ \\
\hline \multirow[t]{2}{*}{ Sign } & & $*$ & ns & ns & ns & ns \\
\hline & $\mathrm{C}$ & $40.7 \pm 0.9 b$ & $3018 \pm 80$ & $2119 \pm 85$ & $1771 \pm 136 b$ & $0.84 \pm 0.09$ \\
\hline \multirow[t]{2}{*}{ Sangiovese } & $\mathrm{D}$ & $39.0 \pm 2.1 \mathrm{ab}$ & $2821 \pm 108$ & $2083 \pm 120$ & $1513 \pm 81 \mathrm{ab}$ & $0.73 \pm 0.01$ \\
\hline & $\mathrm{E}$ & $35.8 \pm 2.0 \mathrm{a}$ & $2607 \pm 307$ & $1824 \pm 324$ & $1404 \pm 174 a$ & $0.78 \pm 0.07$ \\
\hline \multirow[t]{2}{*}{ Sign } & & $*$ & $\mathrm{~ns}$ & $\mathrm{~ns}$ & $*$ & ns \\
\hline & $\mathrm{D}$ & $28.9 \pm 2.5$ & $2133 \pm 164$ & $1133 \pm 71$ & $1023 \pm 49$ & $0.90 \pm 0.03$ \\
\hline \multirow[t]{2}{*}{ Sauvignon Blanc } & $\mathrm{E}$ & $26.0 \pm 2.3$ & $2091 \pm 136$ & $1112 \pm 123$ & $1008 \pm 64$ & $0.91 \pm 0.07$ \\
\hline & $\mathrm{F}$ & $27.2 \pm 4.6$ & $2071 \pm 270$ & $988 \pm 19$ & $1019 \pm 121$ & $1.03 \pm 0.14$ \\
\hline \multirow[t]{2}{*}{ Sign } & & ns & ns & ns & $\mathrm{ns}$ & ns \\
\hline & $\mathrm{D}$ & $36.2 \pm 4.5$ & $3010 \pm 382$ & $2002 \pm 200 b$ & $1562 \pm 167 b$ & $0.78 \pm 0.05$ \\
\hline \multirow[t]{2}{*}{ Syrah } & $\mathrm{E}$ & $34.8 \pm 4.9$ & $2839 \pm 380$ & $1941 \pm 82 b$ & $1592 \pm 143 b$ & $0.82 \pm 0.04$ \\
\hline & $\mathrm{F}$ & $26.8 \pm 3.8$ & $2225 \pm 259$ & $1583 \pm 159 a$ & $1158 \pm 110 \mathrm{a}$ & $0.73 \pm 0.02$ \\
\hline Sign & & ns & ns & $*$ & $*$ & ns \\
\hline
\end{tabular}

Data are expressed as mean value \pm standard deviation $(n=3)$. Different Latin letters within the same column indicate significant differences $\left({ }^{\mathrm{a}}\right)$ among density classes according to the Tukey-b test $(p<0.05)$. Sign ${ }^{\mathrm{a}}: *$ and ns indicate significance at $p<0.05$ and not significant, respectively. $\mathrm{A}_{280}=$ absorbance measured at $280 \mathrm{~nm}, \mathrm{TF}=$ total flavonoids, $\mathrm{PRO}=$ proanthocyanidins, $\mathrm{FRV}$ = flavanols reactive to vanillin. $\mathrm{C}=1088 \mathrm{~kg} / \mathrm{m}^{3}, \mathrm{D}=1094 \mathrm{~kg} / \mathrm{m}^{3}, \mathrm{E}=1100 \mathrm{~kg} / \mathrm{m}^{3}, \mathrm{~F}=1107 \mathrm{~kg} / \mathrm{m}^{3}$.

Noir seeds were characterised by the highest values of these spectrophotometric indices, although the Mourvèdre cultivar had also a high extractable content of PRO in the seeds.

Regarding extractable monomeric and dimeric flavanols (Table 4), the Cinsault cultivar was characterised by the lowest content in the seeds of $(+)$-catechin $(\mathrm{CA})$ and procyanidin $\mathrm{B}_{1}$, although low amounts of other compounds like (-)-epicatechin gallate (ECG) were also found. Grenache seeds accounted for the lowest amount of (-)-epicatechin (EC) and $\mathrm{ECG}$, although low contents of $\mathrm{CA}$ and procyanidins $\mathrm{B}_{1}$ and $\mathrm{B}_{2}$ were also observed. Malvasia Bianca seeds were characterised by the lowest amount of procyanidin $\mathrm{B}_{2}$ and by a comparatively low content of EC and ECG. Erbaluce and Avaná seeds had also low contents of EC. Barbera, Sauvignon Blanc and Moscato Bianco seeds contained low concentrations of ECG, procyanidin $\mathrm{B}_{1}$ and $\mathrm{B}_{2}$, respectively. In contrast, the Pinot Noir cultivar showed the highest content of CA in the seeds, whereas Petit Verdot seeds were the richest in ECG and accounted for a high amount of procyanidin $B_{1}$. Furthermore, the Pinot Noir and Petit Rouge cultivars had the highest content of EC and procyanidins B and $\mathrm{B}_{2}$ in the seeds, but also showed quite high amounts of ECG. Pignolo Spano seeds were rich in EC and procyanidin $\mathrm{B}_{2}$, whereas Merlot, Freisa and Mourvèdre seeds contained high amounts of ECG. The presence of gallic acid was higher in Brachetto d'Acqui, Merlot and Albarossa seeds.

The effect of berry density on the extractable content of gallic acid and monomeric and dimeric flavanols in the seeds of the wine grape varieties studied was quite small at harvest, and few significant differences were found in these contents among density classes when each variety was evaluated individually. However, a decreasing trend was mostly observed, as also occurred for the spectrophotometric indices. This agreed with the increased association of seed flavanols with cell-wall components resulting in the gradual decline of the extractable amount as ripening advanced (Kennedy et al., 2000a, 2000b; Downey et al., 2003; Cadot et al., 2006; Ferrer-Gallego et al., 2010; Lorrain et al., 2011; Obreque-Slier et al., 2012). 


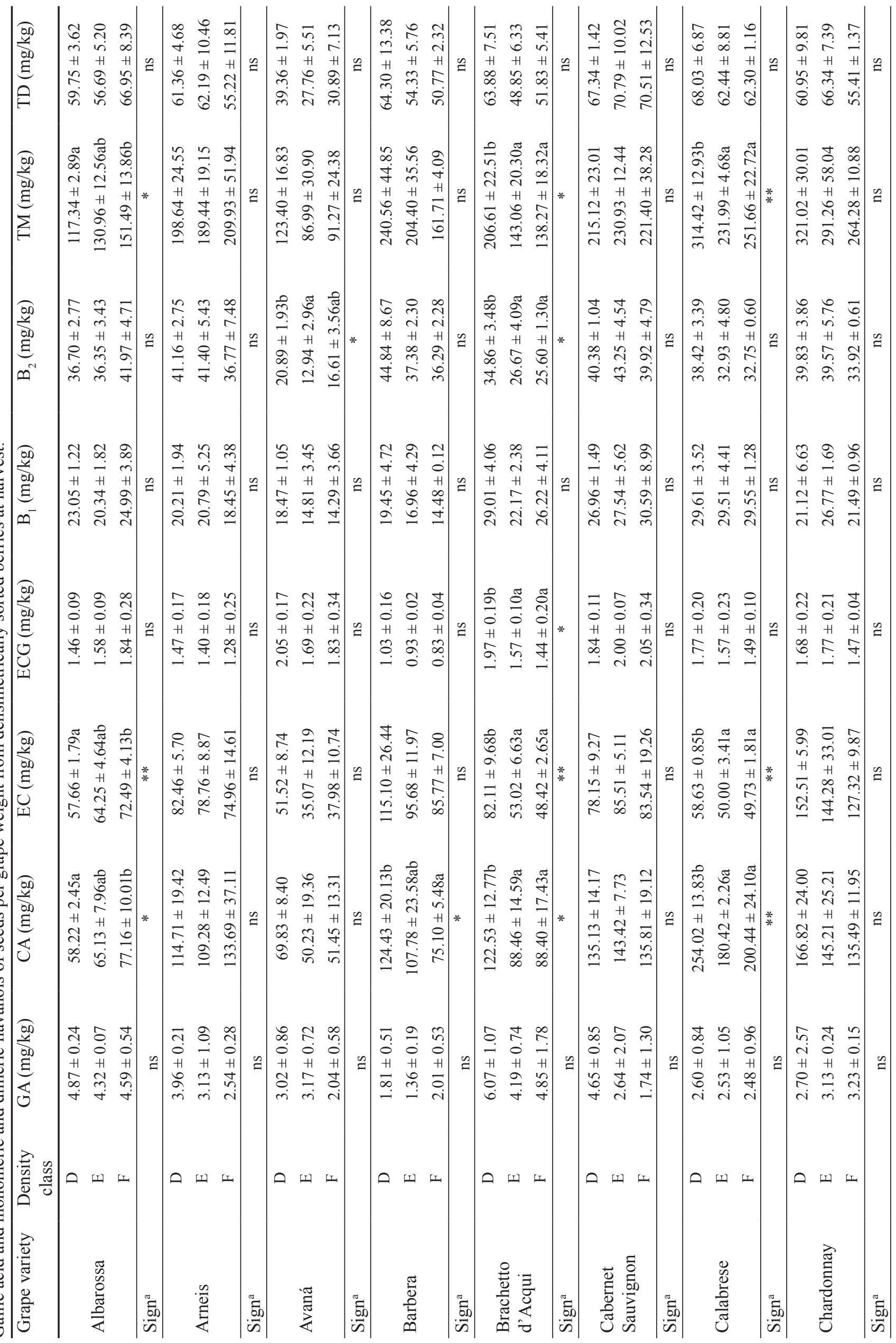




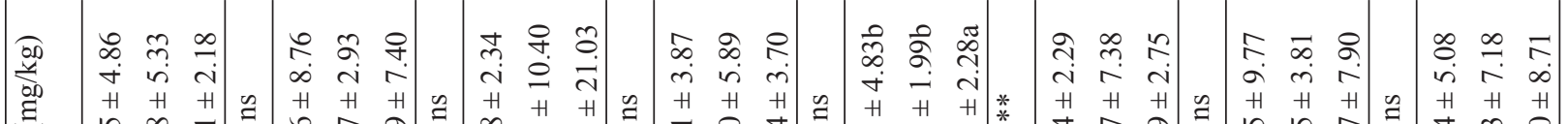

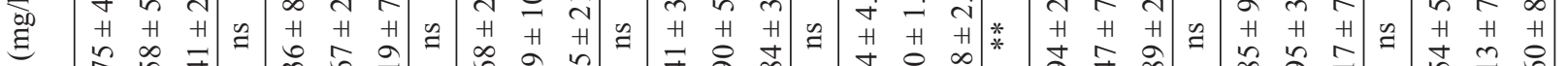

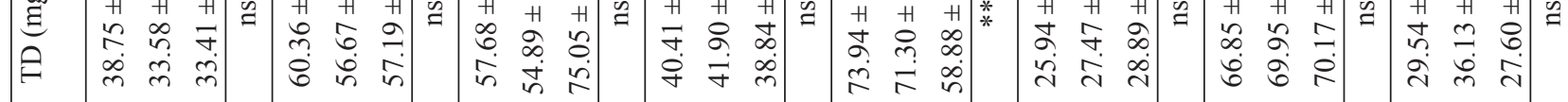

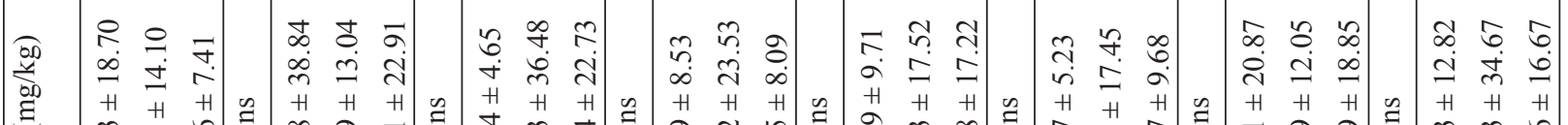

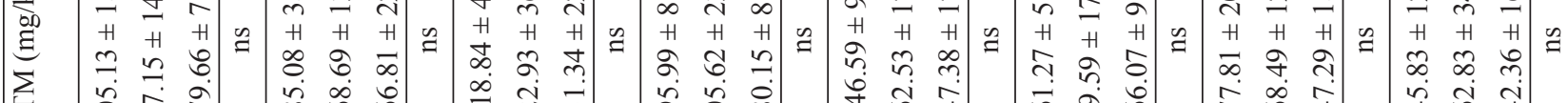

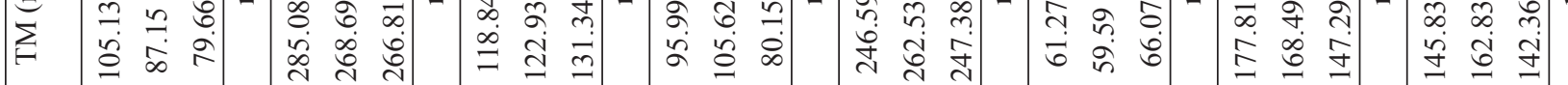

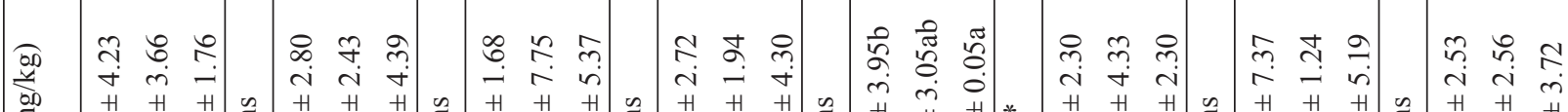

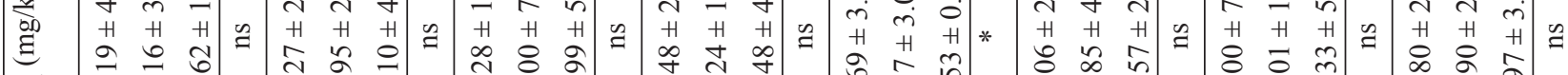

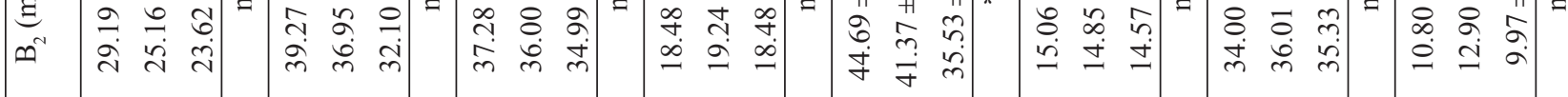

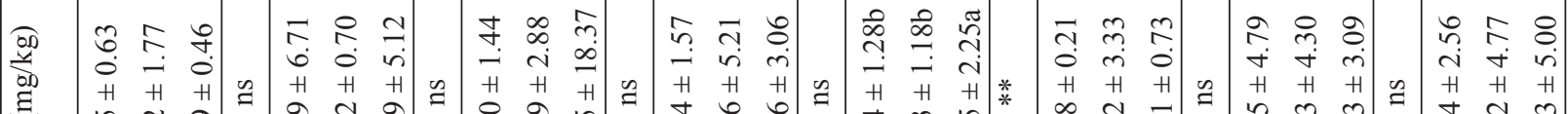

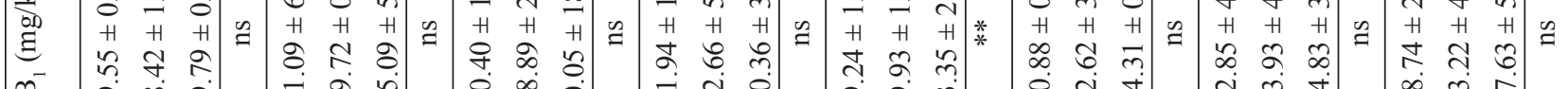

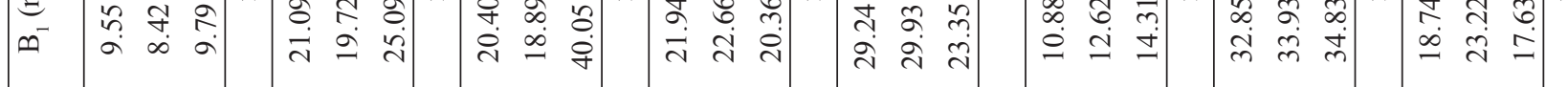

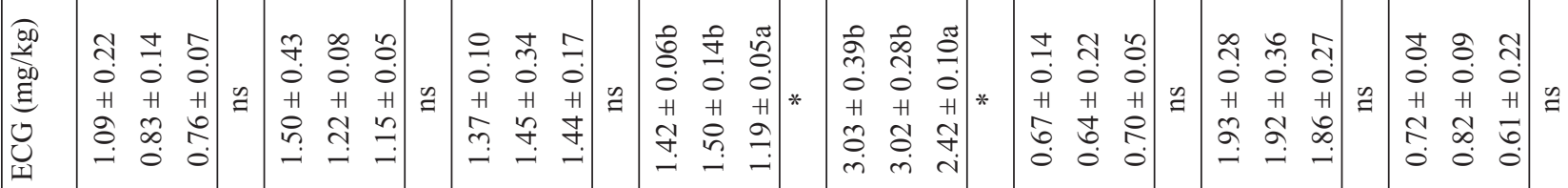

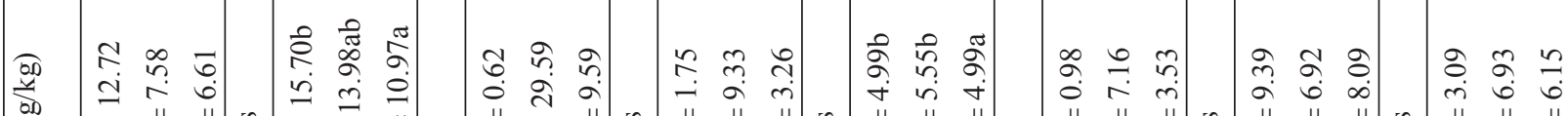

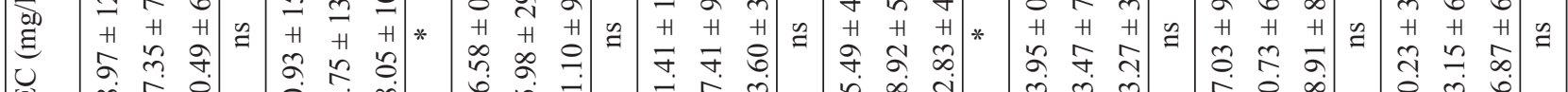

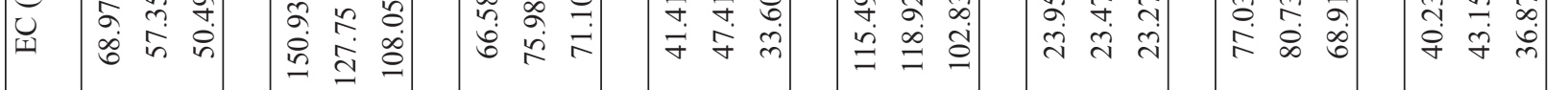

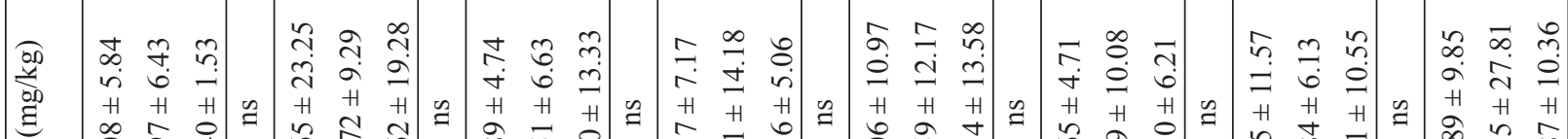

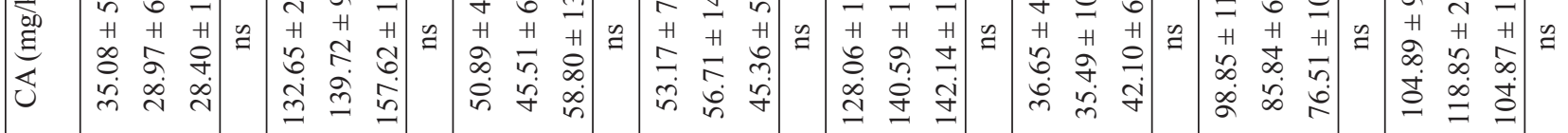

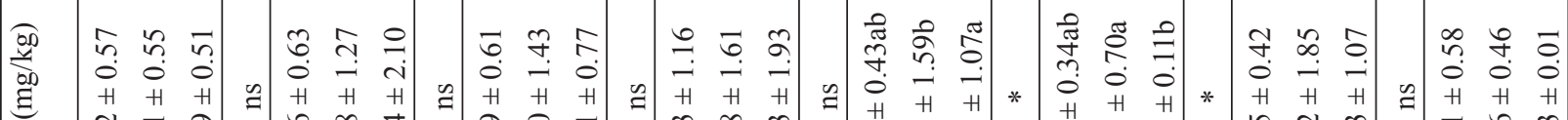

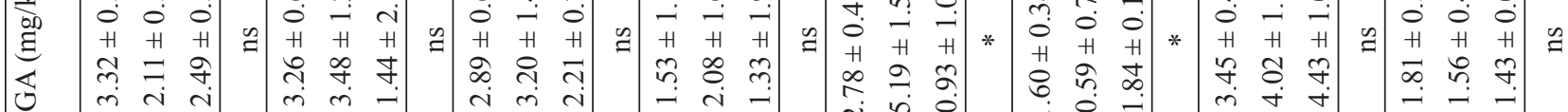

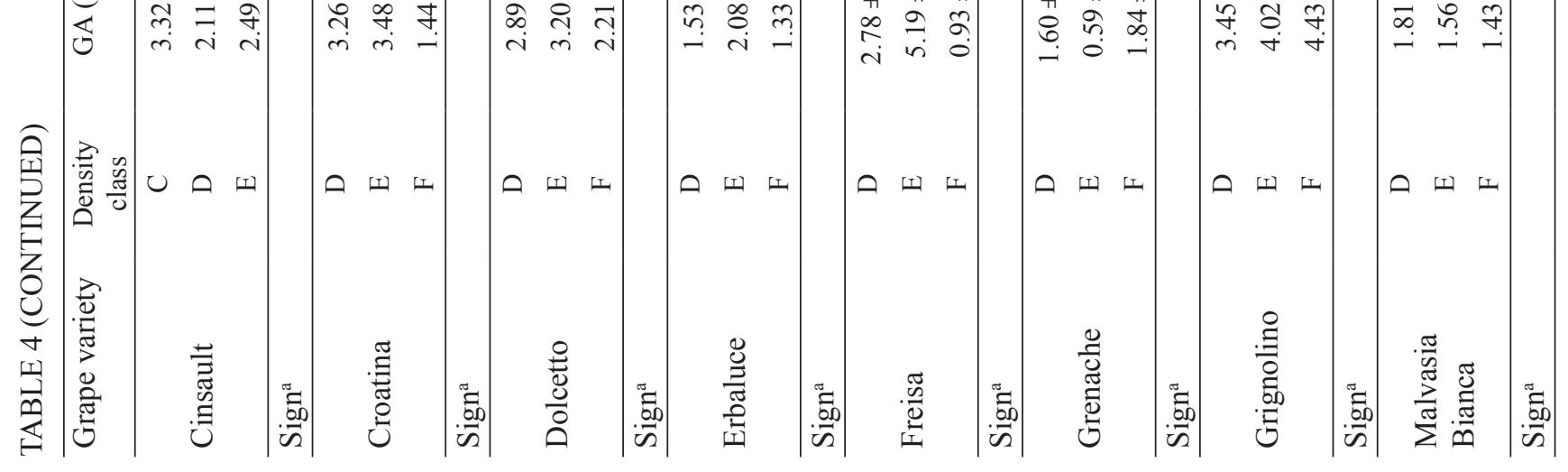




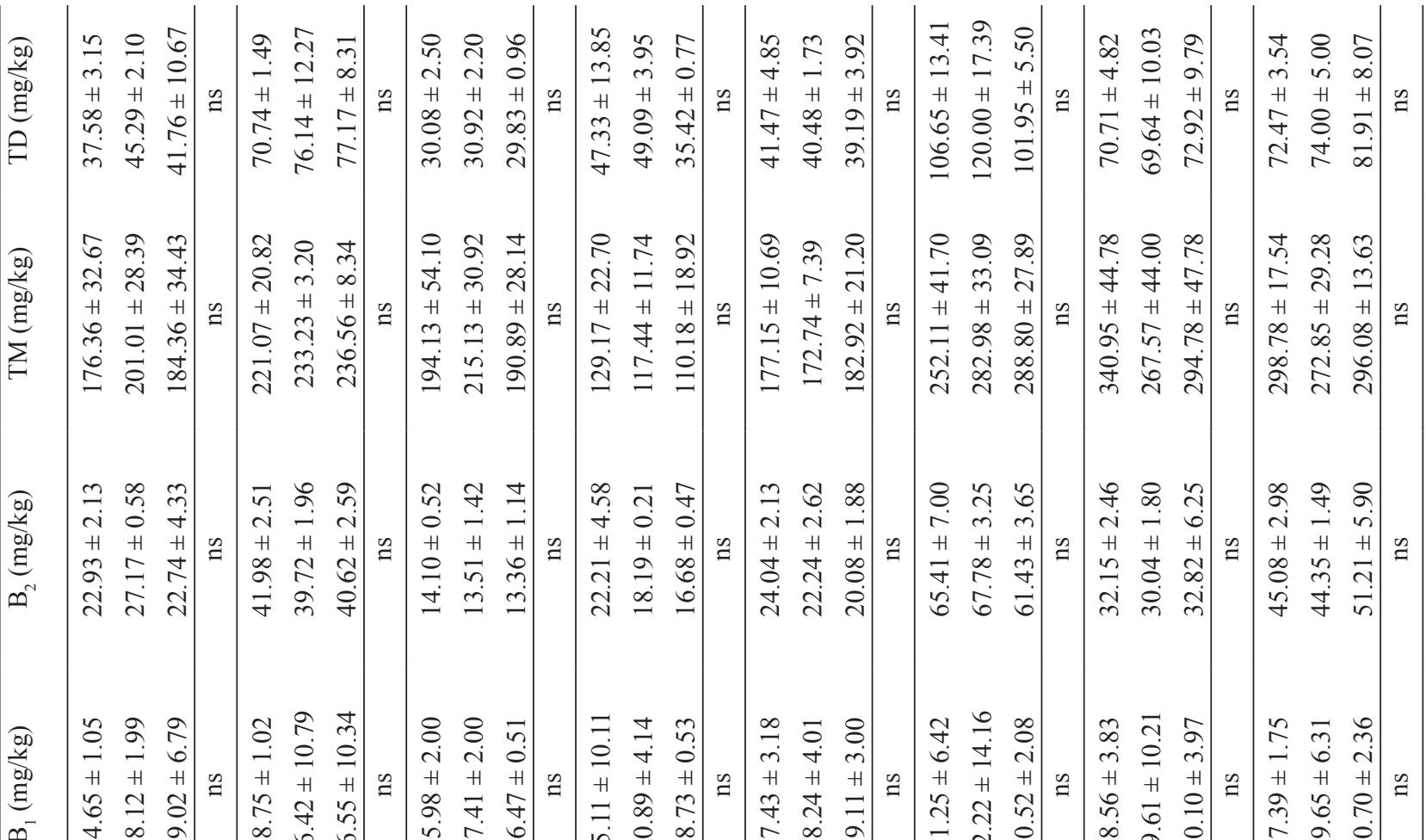

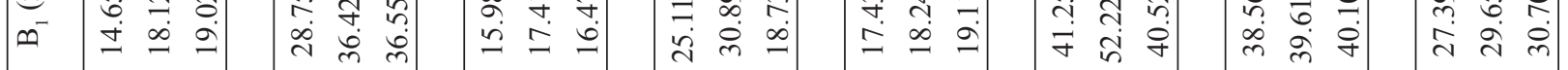

可

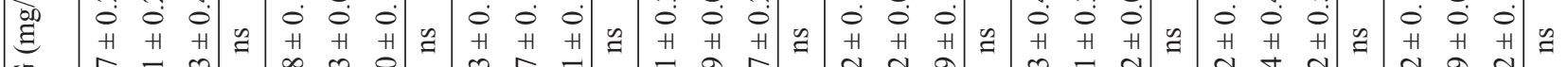

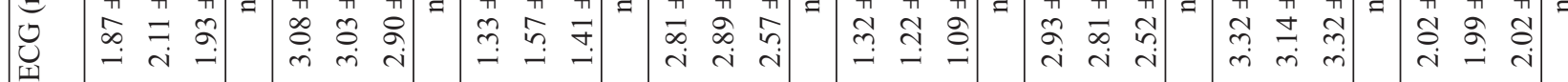

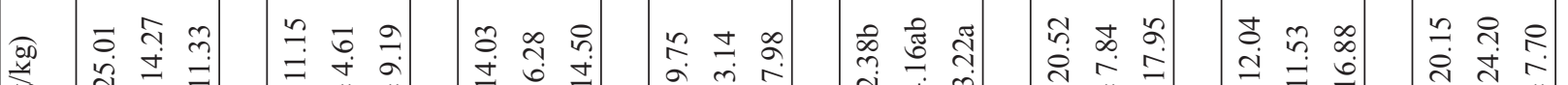

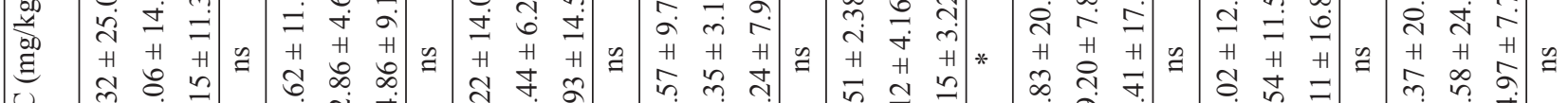

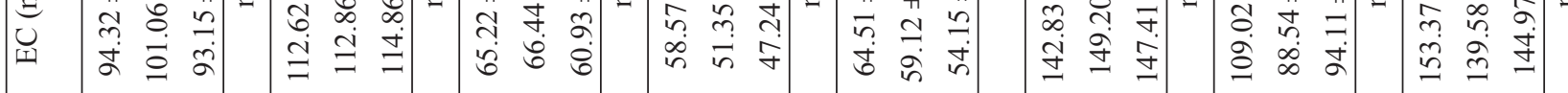

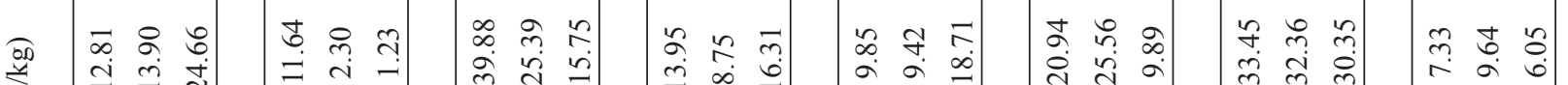

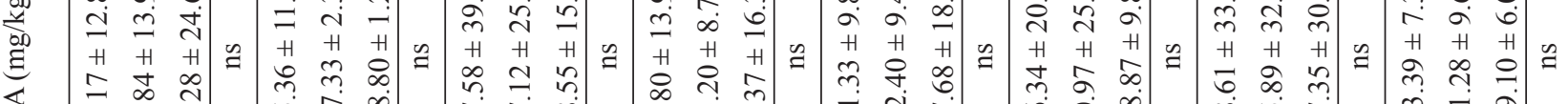

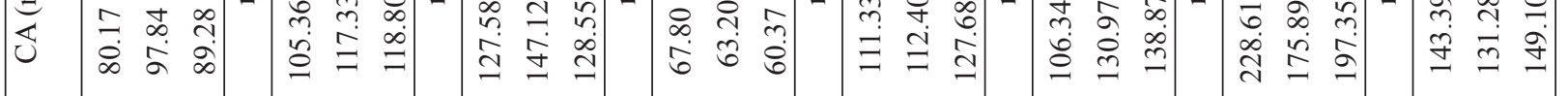

का

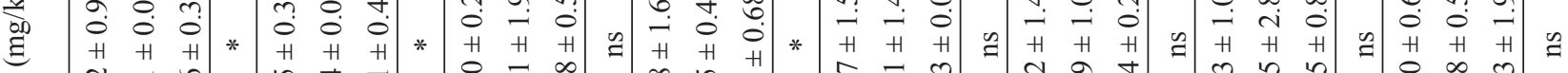

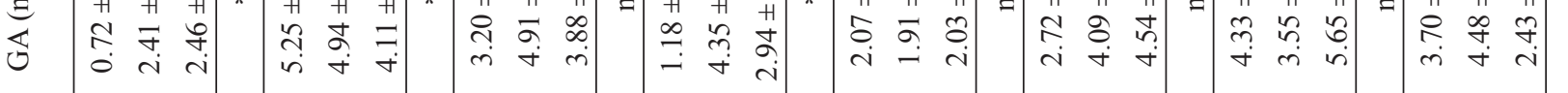

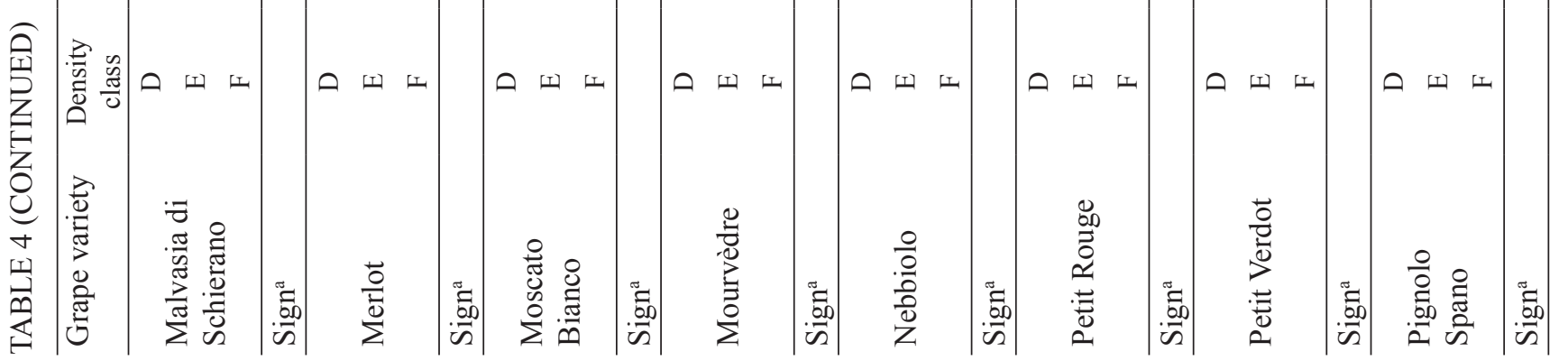




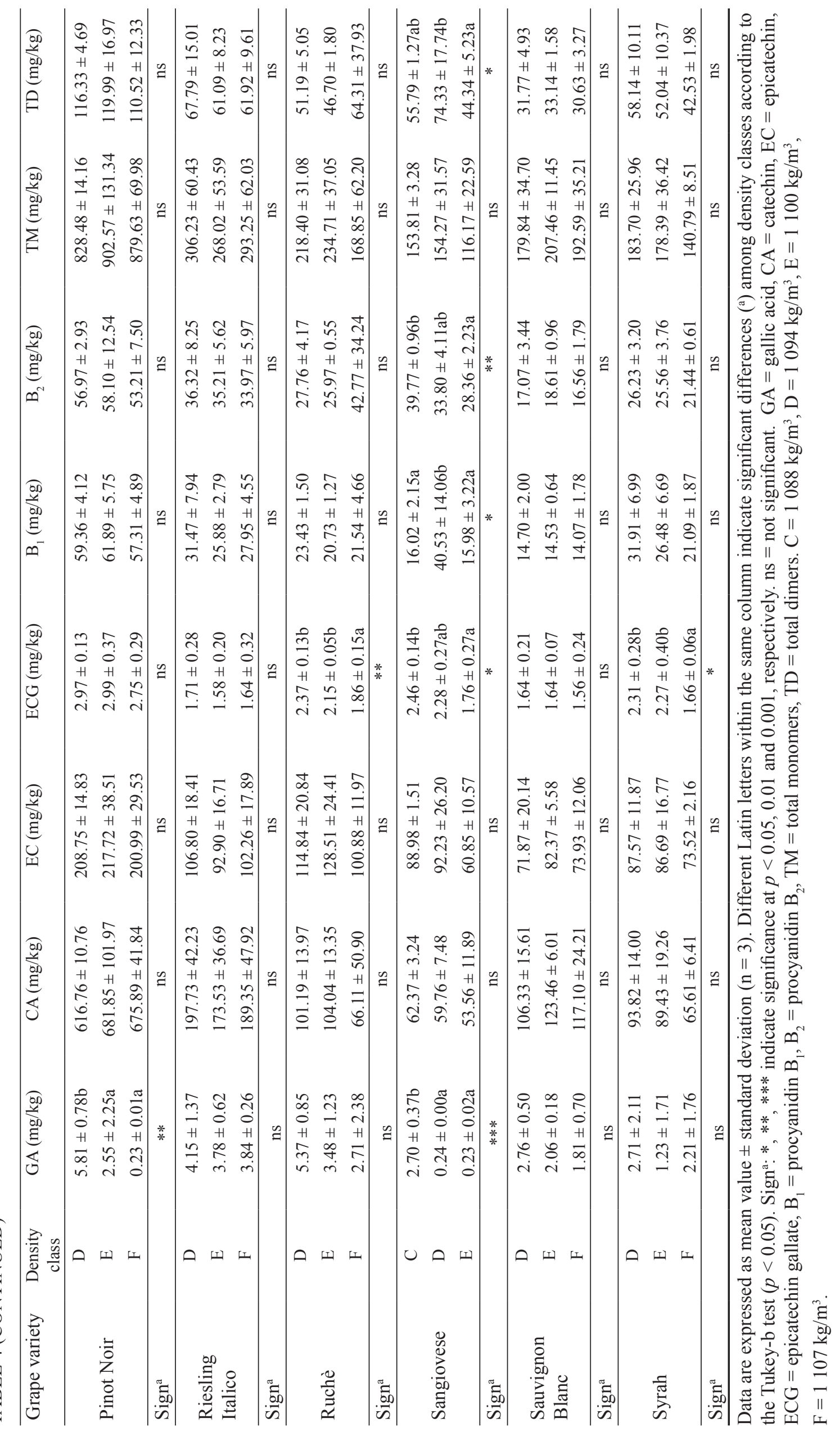


The next aim was the establishment of robust relationships between the instrumental mechanical properties of grape seeds (Table 2) and the phenolic composition determined by the reference chemical methods. A correlation study was performed using the reference values of the extractable content of phenolic compounds in the seeds, expressed per grape weight (Tables 3 and 4) and seed weight. When all of the wine grape varieties and density classes studied were used, the performance of the texture parameters of the seeds as predictors of the extractable content of phenolic compounds was quite poor, with significant correlation coefficients ranging from $0.121(p<0.05)$ to $0.363(p<0.001)$. The weakness of these relationships led to a separate correlation study for each cluster established according to seed hardness (Fig. 2). Table 5 shows the variation range of the spectrophotometric indices and the extractable content of gallic acid and flavanolic monomers and dimers in the seeds, expressed per grape and seed weight, using all of the varieties included in each cluster and density class studied. Likewise, Table 6 reports the significant correlation coefficients (R) between the mechanical and chemical parameters of the seeds summarised in Table 5. The most significant and strongest correlations were found for the varieties belonging to cluster 1 (softest seeds), the coefficients lying between 0.504 and $0.640(p<0.001)$ for the correlations of $\mathrm{A}_{280}, \mathrm{TF}, \mathrm{FRV}$ and TD expressed per grape weight with $\mathrm{F}_{\mathrm{s}}$ and $\mathrm{W}_{\mathrm{s}}$, and those of PRO, CA, EC, ECG, procyanidin $\mathrm{B}_{1}$ and TM expressed per grape weight or FRV and TD expressed per seed weight with $\mathrm{W}_{\mathrm{s}}$. It is important to take into account that, although most of the chemical parameters showed significant correlations in cluster 1 with the break force, break energy and deformation index of the seeds, no significant relationship was found with the resistance of the seed to axial deformation. Instead, different chemical parameters were significantly correlated with $\mathrm{E}_{\mathrm{s}}(p<0.05)$ in clusters 2 and 3, particularly if they were expressed per seed weight. Significant correlations were also observed in clusters 2 and 3 between the chemical and mechanical parameters of the seeds, but the coefficients were too small ( $\mathrm{R}<0.500)$, except for the relationship $(p<0.001)$ between PRO expressed per seed weight and $\mathrm{E}_{\mathrm{s}}(\mathrm{R}=-0.525)$, FRV/PRO and DI $(\mathrm{R}=-0.508), \mathrm{GA}$ expressed per seed weight and $\mathrm{E}_{\mathrm{s}}(\mathrm{R}=-0.538)$, and $\mathrm{ECG}$, also expressed per seed weight, and $\mathrm{W}_{\mathrm{s}}(\mathrm{R}=0.504)$ in cluster 2 .

This work confirmed the relationship reported in a preliminary study performed on Cabernet Sauvignon seeds, where the strongest and most significant correlation for the $A_{280}$ values per seed weight measured in the extracts obtained after seed treatment with a wine-like solution was found with $\mathrm{E}_{\mathrm{s}}$ (Rolle et al., 2012a). In the present study, wine grape varieties that belong to cluster 2, including Cabernet Sauvignon, showed a significant correlation between these two parameters, but the correlation coefficient improved $(\mathrm{R}=0.406$ instead of $0.190, \mathrm{p}<0.01)$. In Merlot seeds, the only significant correlation reported between mechanical and chemical parameters corresponded to the relationship of the extractable content of FRV expressed per grape weight with $\mathrm{F}_{\mathrm{s}}(\mathrm{R}=0.452, \mathrm{p}<0.05)$ (Torchio et al., 2012). In the present work, this later chemical parameter was the only spectrophotometric index that showed significant correlations for the Merlot cultivar (as it belongs to cluster 3) when the results were expressed per grape weight, although not with $\mathrm{F}_{\mathrm{s}}$ but DI. However, the extractable content of FRV and $F_{s}$ were correlated significantly when the results were expressed per seed weight $(\mathrm{R}=0.205, \mathrm{p}<0.05)$.

Univariate linear regression calibration models were only constructed for the most significant and strongest correlations between the phenolic composition of the seeds, determined by the reference chemical methods,

TABLE 5

Spectrophotometric indices, gallic acid and monomeric and dimeric flavanols of seeds per grape and seed weight for all varieties belonging to the clusters defined by the seed hardness and density classes.

\begin{tabular}{|c|c|c|c|c|c|c|c|c|c|c|c|c|}
\hline \multirow{2}{*}{$\begin{array}{l}\text { Chemical } \\
\text { parameter }\end{array}$} & \multicolumn{4}{|c|}{ Cluster 1} & \multicolumn{4}{|c|}{ Cluster 2} & \multicolumn{4}{|c|}{ Cluster 3} \\
\hline & Min & Max & Mean & SD & Min & Max & Mean & SD & Min & Max & Mean & SD \\
\hline \multicolumn{13}{|c|}{ mg/kg grape } \\
\hline $\mathrm{A}_{280}$ & 16.8 & 76.5 & 39.2 & 11.6 & 20.2 & 59.8 & 34.2 & 10.2 & 13.6 & 75.1 & 40.4 & 12.6 \\
\hline $\mathrm{TF}$ & 1319 & 5146 & 3015 & 798 & 1525 & 4483 & 2587 & 763 & 1106 & 5368 & 3016 & 907 \\
\hline PRO & 1004 & 3435 & 1984 & 549 & 933 & 3339 & 1589 & 674 & 650 & 3707 & 2010 & 646 \\
\hline FRV & 589 & 3293 & 1614 & 550 & 768 & 2179 & 1334 & 422 & 508 & 2946 & 1600 & 478 \\
\hline GA & 0.14 & 7.19 & 3.15 & 1.75 & 0.21 & 5.56 & 2.48 & 1.23 & 0.22 & 6.93 & 2.95 & 1.59 \\
\hline CA & 7.56 & 793.41 & 137.41 & 158.34 & 28.45 & 173.93 & 111.34 & 36.07 & 22.89 & 269.93 & 119.06 & 54.21 \\
\hline $\mathrm{EC}$ & 16.62 & 244.13 & 88.16 & 48.17 & 21.45 & 160.53 & 85.55 & 32.82 & 30.01 & 175.38 & 86.53 & 36.89 \\
\hline ECG & 0.47 & 3.41 & 1.73 & 0.55 & 0.79 & 2.26 & 1.53 & 0.43 & 0.37 & 3.71 & 2.05 & 0.88 \\
\hline $\mathrm{B}_{1}$ & 9.66 & 68.50 & 25.89 & 12.26 & 11.13 & 40.61 & 19.52 & 6.45 & 6.82 & 68.09 & 26.72 & 11.27 \\
\hline $\mathrm{B}_{2}$ & 9.90 & 82.29 & 30.44 & 12.85 & 10.30 & 54.53 & 30.20 & 11.68 & 5.80 & 71.51 & 34.30 & 14.14 \\
\hline TM & 43.03 & 1032.33 & 227.30 & 200.45 & 51.34 & 315.31 & 198.42 & 64.05 & 71.12 & 390.18 & 207.64 & 73.49 \\
\hline TD & 19.55 & 135.20 & 56.32 & 23.31 & 21.43 & 82.69 & 49.72 & 16.59 & 17.74 & 139.60 & 61.01 & 22.62 \\
\hline
\end{tabular}


TABLE 5 (CONTINUED)

\begin{tabular}{|c|c|c|c|c|c|c|c|c|c|c|c|c|}
\hline \multirow{2}{*}{$\begin{array}{l}\text { Chemical } \\
\text { parameter }\end{array}$} & \multicolumn{4}{|c|}{ Cluster 1} & \multicolumn{4}{|c|}{ Cluster 2} & \multicolumn{4}{|c|}{ Cluster 3} \\
\hline & Min & Max & Mean & SD & Min & Max & Mean & SD & Min & Max & Mean & SD \\
\hline \multicolumn{13}{|c|}{$\mathrm{mg} / \mathrm{g}$ seed } \\
\hline $\mathrm{A}_{280}$ & 0.77 & 1.58 & 1.09 & 0.21 & 0.76 & 1.36 & 1.04 & 0.15 & 0.67 & 1.43 & 1.03 & 0.16 \\
\hline $\mathrm{TF}$ & 59.7 & 116.2 & 84.2 & 13.4 & 64.6 & 98.0 & 78.3 & 9.9 & 55.5 & 100.9 & 76.9 & 11.5 \\
\hline PRO & 39.1 & 82.2 & 55.3 & 8.9 & 31.0 & 61.0 & 46.8 & 8.8 & 36.2 & 79.5 & 51.1 & 9.4 \\
\hline FRV & 27.8 & 74.9 & 44.7 & 10.2 & 30.4 & 54.6 & 40.2 & 5.8 & 20.7 & 54.9 & 41.1 & 7.8 \\
\hline FRV/PRO & 0.59 & 1.07 & 0.81 & 0.11 & 0.65 & 1.19 & 0.87 & 0.12 & 0.57 & 1.21 & 0.81 & 0.13 \\
\hline GA & 0.00 & 0.24 & 0.09 & 0.05 & 0.01 & 0.15 & 0.08 & 0.04 & 0.01 & 0.18 & 0.08 & 0.04 \\
\hline $\mathrm{CA}$ & 0.24 & 17.91 & 3.68 & 3.62 & 1.27 & 4.89 & 3.40 & 0.95 & 1.06 & 8.64 & 3.09 & 1.52 \\
\hline $\mathrm{EC}$ & 0.79 & 5.86 & 2.44 & 1.17 & 0.96 & 4.48 & 2.64 & 0.97 & 1.02 & 4.61 & 2.25 & 0.86 \\
\hline ECG & 0.02 & 0.09 & 0.05 & 0.01 & 0.02 & 0.09 & 0.05 & 0.02 & 0.01 & 0.09 & 0.05 & 0.02 \\
\hline $\mathrm{B}_{1}$ & 0.38 & 1.58 & 0.71 & 0.25 & 0.42 & 0.86 & 0.59 & 0.10 & 0.31 & 1.61 & 0.69 & 0.25 \\
\hline $\mathrm{B}_{2}$ & 0.43 & 2.66 & 0.83 & 0.27 & 0.46 & 1.59 & 0.91 & 0.29 & 0.21 & 1.69 & 0.91 & 0.38 \\
\hline $\mathrm{TM}$ & 2.04 & 23.31 & 6.17 & 4.55 & 2.29 & 9.03 & 6.09 & 1.79 & 2.16 & 10.51 & 5.39 & 1.71 \\
\hline TD & 0.92 & 3.48 & 1.54 & 0.45 & 0.96 & 2.32 & 1.50 & 0.33 & 0.64 & 3.30 & 1.59 & 0.54 \\
\hline
\end{tabular}

$\mathrm{n}=117$ for cluster $1, \mathrm{n}=45$ for cluster $2, \mathrm{n}=108$ for cluster $3 . \mathrm{SD}=$ standard deviation. $\mathrm{A}_{280}=$ absorbance measured at $280 \mathrm{~nm}, \mathrm{TF}=$ total flavonoids, $\mathrm{PRO}=$ proanthocyanidins, $\mathrm{FRV}=$ flavanols reactive to vanillin, $\mathrm{GA}=$ gallic acid, $\mathrm{CA}=$ catechin, $\mathrm{EC}=$ epicatechin, $\mathrm{ECG}=$ epicatechin gallate, $\mathrm{B}_{1}=$ procyanidin $\mathrm{B}_{1}, \mathrm{~B}_{2}=$ procyanidin $\mathrm{B}_{2}, \mathrm{TM}=$ total monomers, $\mathrm{TD}=$ total dimers.

TABLE 6

Significant Pearson's correlation coefficients between instrumental mechanical properties and chemical parameters related to the phenolic composition of seeds for varieties belonging to the clusters defined by seed hardness.

\begin{tabular}{|c|c|c|c|c|c|c|c|c|c|c|c|}
\hline \multirow[b]{2}{*}{ Parameter } & \multicolumn{3}{|c|}{ Cluster 1} & \multicolumn{4}{|c|}{ Cluster 2} & \multicolumn{4}{|c|}{ Cluster 3} \\
\hline & $\begin{array}{l}\mathrm{F}_{\mathrm{s}} \\
(\mathrm{N})\end{array}$ & $\begin{array}{c}\mathrm{W}_{\mathrm{s}} \\
(\mathrm{mJ})\end{array}$ & $\begin{array}{l}\mathrm{DI}_{\mathrm{s}} \\
(\%)\end{array}$ & $\begin{array}{c}\mathrm{F}_{\mathrm{s}} \\
(\mathrm{N})\end{array}$ & $\begin{array}{c}\mathrm{W}_{\mathrm{s}} \\
(\mathrm{mJ})\end{array}$ & $\begin{array}{c}\mathrm{E}_{\mathrm{s}} \\
(\mathrm{N} / \mathrm{mm})\end{array}$ & $\begin{array}{l}\mathrm{DI}_{\mathrm{s}} \\
(\%) \\
\end{array}$ & $\begin{array}{c}\mathrm{F}_{\mathrm{s}} \\
(\mathrm{N})\end{array}$ & $\begin{array}{c}\mathrm{W}_{\mathrm{s}} \\
(\mathrm{mJ})\end{array}$ & $\begin{array}{c}\mathrm{E}_{\mathrm{s}} \\
(\mathrm{N} / \mathrm{mm})\end{array}$ & $\mathrm{DI}_{\mathrm{s}}(\%)$ \\
\hline \multicolumn{12}{|c|}{ mg/kg grape } \\
\hline $\mathrm{A}_{280}$ & $0.521^{* * *}$ & $0.639^{* * *}$ & $0.408^{* * *}$ & & & & & & & & \\
\hline TF & $0.505^{* * *}$ & $0.602^{* * *}$ & $0.382^{* * *}$ & & & & & & & & \\
\hline PRO & $0.450^{* * *}$ & $0.529^{* * *}$ & $0.392^{* * *}$ & & & & & & & & \\
\hline FRV & $0.527^{* * *}$ & $0.640^{* * *}$ & $0.403^{* * *}$ & & & & & & & & $-0.229^{*}$ \\
\hline GA & & $0.190^{*}$ & & $-0.297^{*}$ & & $-0.368^{*}$ & & & & $0.274^{* *}$ & \\
\hline $\mathrm{CA}$ & $0.362^{* * *}$ & $0.521^{* * *}$ & $0.419^{* * *}$ & & & & & & $-0.238^{*}$ & $0.224^{*}$ & \\
\hline $\mathrm{EC}$ & $0.416^{* * *}$ & $0.525^{* * *}$ & $0.359^{* * *}$ & & $-0.342^{*}$ & & & & & & \\
\hline ECG & $0.470^{* * *}$ & $0.574^{* * *}$ & $0.308^{* * *}$ & & $0.327^{*}$ & $-0.311^{*}$ & & & $0.196^{*}$ & & \\
\hline $\mathrm{B}_{1}$ & $0.477^{* * *}$ & $0.563^{* * *}$ & $0.331^{* * *}$ & & & & & & & & $-0.215^{*}$ \\
\hline $\mathrm{B}_{2}$ & $0.463^{* * *}$ & $0.496^{* * *}$ & $0.283^{* *}$ & & $-0.337^{*}$ & & & & & $0.216^{*}$ & \\
\hline TM & $0.387^{* * *}$ & $0.539^{* * *}$ & $0.418^{* * *}$ & & $-0.330^{*}$ & & & & & $0.219^{*}$ & \\
\hline TD & $0.507^{* * *}$ & $0.570^{* * *}$ & $0.331^{* * *}$ & & $-0.297^{*}$ & & & & & & \\
\hline \multicolumn{12}{|c|}{$\mathrm{mg} / \mathrm{g}$ seed } \\
\hline $\mathrm{A}_{280}$ & $0.293^{* *}$ & $0.460^{* * *}$ & $0.343^{* * *}$ & & & $-0.406^{* *}$ & & & & & \\
\hline $\mathrm{TF}$ & $0.261^{* *}$ & $0.423^{* * *}$ & $0.336^{* * *}$ & & & $-0.431^{* *}$ & & & & & \\
\hline PRO & $0.184^{*}$ & $0.334^{* * *}$ & $0.394^{* * *}$ & $-0.342^{*}$ & & $-0.525^{* * *}$ & $0.446^{* *}$ & & & & $-0.212^{*}$ \\
\hline FRV & $0.390^{* * *}$ & $0.543^{* * *}$ & $0.367^{* * *}$ & & & $-0.372^{*}$ & & $-0.205^{*}$ & $-0.318^{* * *}$ & & $-0.374^{* * *}$ \\
\hline
\end{tabular}




\begin{tabular}{|c|c|c|c|c|c|c|c|c|c|c|c|}
\hline \multirow[b]{2}{*}{ Parameter } & \multicolumn{3}{|c|}{ Cluster 1} & \multicolumn{4}{|c|}{ Cluster 2} & \multicolumn{4}{|c|}{ Cluster 3} \\
\hline & $\begin{array}{c}\mathrm{F}_{\mathrm{s}} \\
(\mathrm{N})\end{array}$ & $\begin{array}{c}\mathrm{W}_{\mathrm{s}} \\
(\mathrm{mJ})\end{array}$ & $\begin{array}{l}\mathrm{DI}_{\mathrm{s}} \\
(\%)\end{array}$ & $\begin{array}{l}\mathrm{F}_{\mathrm{s}} \\
(\mathrm{N})\end{array}$ & $\begin{array}{c}\mathrm{W}_{\mathrm{s}} \\
(\mathrm{mJ})\end{array}$ & $\begin{array}{c}\mathrm{E}_{\mathrm{s}} \\
(\mathrm{N} / \mathrm{mm})\end{array}$ & $\begin{array}{l}\mathrm{DI}_{\mathrm{s}} \\
(\%)\end{array}$ & $\begin{array}{c}\mathrm{F}_{\mathrm{s}} \\
(\mathrm{N})\end{array}$ & $\begin{array}{c}\mathrm{W}_{\mathrm{s}} \\
(\mathrm{mJ})\end{array}$ & $\begin{array}{c}\mathrm{E}_{\mathrm{s}} \\
(\mathrm{N} / \mathrm{mm})\end{array}$ & $\begin{array}{l}\mathrm{DI}_{\mathrm{s}} \\
(\%)\end{array}$ \\
\hline FRV/PRO & $0.406^{* * *}$ & $0.477^{* * *}$ & & & & $0.375^{*}$ & $-0.508^{* * *}$ & & $-0.325^{* * *}$ & $0.252^{* *}$ & $-0.274^{* *}$ \\
\hline GA & & & & $-0.361^{*}$ & & $-0.538^{* * *}$ & & & & $0.283^{* *}$ & \\
\hline $\mathrm{CA}$ & $0.308^{* * *}$ & $0.472^{* * *}$ & $0.391^{* * *}$ & & & $0.315^{*}$ & $-0.329^{*}$ & $-0.204^{*}$ & $-0.379^{* * *}$ & $0.204^{*}$ & $-0.208^{*}$ \\
\hline $\mathrm{EC}$ & $0.296^{* *}$ & $0.400^{* * *}$ & $0.278^{* *}$ & & & & & & & & \\
\hline ECG & $0.200^{*}$ & $0.300^{* *}$ & & & $0.504^{* * *}$ & $-0.413^{* *}$ & & & & & \\
\hline $\mathrm{B}_{1}$ & $0.374^{* * *}$ & $0.473^{* * *}$ & $0.284^{* *}$ & & & & $0.305^{*}$ & & $-0.265^{* *}$ & & $-0.306^{* *}$ \\
\hline $\mathrm{B}_{2}$ & $0.371^{* * *}$ & $0.400^{* * * *}$ & $0.226^{*}$ & & & $0.326^{*}$ & & $0.220^{*}$ & & $0.243^{*}$ & \\
\hline $\mathrm{TM}$ & $0.322^{* * *}$ & $0.479^{* * *}$ & $0.383^{* * *}$ & & & $0.316^{*}$ & $-0.327^{*}$ & & $-0.293^{* *}$ & $0.261^{* *}$ & \\
\hline TD & $0.431^{* * *}$ & $0.504^{* * *}$ & $0.294^{* *}$ & & & & & & & $0.235^{*}$ & \\
\hline
\end{tabular}

$\mathrm{n}=117$ for cluster $1, \mathrm{n}=45$ for cluster $2, \mathrm{n}=108$ for cluster 3 . Sign: ${ }^{*},{ }^{* *},{ }^{* * *}$ indicate significance at $p<0.05,0.01$ and 0.001 , respectively. $\mathrm{F}_{\mathrm{s}}=$ seed break force, $\mathrm{W}_{\mathrm{s}}=$ seed break energy, $\mathrm{E}_{\mathrm{s}}=$ resistance of the seed to axial deformation, $\mathrm{DI}=\mathrm{seed}$ deformation index. $\mathrm{A}_{280}=$ absorbance measured at $280 \mathrm{~nm}, \mathrm{TF}=$ total flavonoids, $\mathrm{PRO}=$ proanthocyanidins, $\mathrm{FRV}=$ flavanols reactive to vanillin, $\mathrm{GA}=$ gallic acid, $\mathrm{CA}=$ catechin, $\mathrm{EC}=$ epicatechin, $\mathrm{ECG}=$ epicatechin gallate, $\mathrm{B}_{1}=$ procyanidin $\mathrm{B}_{1}$, $\mathrm{B}_{2}=$ procyanidin $\mathrm{B}_{2}, \mathrm{TM}=$ total monomers, $\mathrm{TD}=$ total dimers.

and the mechanical parameters instrumentally determined $(\mathrm{R}>0.500, p<0.001)$. Table 7 shows that better statistical parameters of calibration corresponded to the relationships between the chemical parameters and the seed break energy than to the break force for wine grape varieties belonging to cluster 1. Regarding these varieties, the relationship of FRV expressed per seed weight with $\mathrm{W}_{\mathrm{s}}$ was statistically the most satisfactory in terms of error $\left(\mathrm{R}_{\mathrm{c}}=0.500, \mathrm{SEC} \%<20\right)$, although the correlation coefficient of calibration was not too good. Other chemical parameters like $\mathrm{A}_{280}, \mathrm{TF}, \mathrm{FRV}$ and ECG expressed per grape weight showed better correlation coefficients of calibration with $\mathrm{W}_{\mathrm{s}}\left(\mathrm{R}_{\mathrm{c}}>0.600\right)$, but the standard error of calibration (SEC) was slightly higher than $20 \%$. On the other hand, the extractable content of TM in the seeds, expressed per grape weight, accounted for extremely high SEC\% values, which may be due to a discontinuous distribution of the CA content $(92.3 \%$ of seed samples contained amounts of CA lower than $245 \mathrm{mg} / \mathrm{kg}$ grape, whereas the amounts in the remaining samples ranged from 593 to $793 \mathrm{mg} / \mathrm{kg}$ grape). When the univariate calibration models were developed for wine grape varieties belonging to cluster 2, the most satisfactory statistical parameters corresponded to the relationships of the spectrophotometric index PRO expressed per seed weight with $\mathrm{E}_{\mathrm{s}}\left(\mathrm{R}_{\mathrm{c}}=0.521\right.$, $\mathrm{SEC} \%<16$ ).

An external validation was performed to assess the robustness of the linear regression calibration models using a sample set that did not belong to the calibration set. The calibration equations obtained were applied to the validation set, and the chemical parameters determined in the seeds by the reference method were compared with those predicted by the calibration models obtained (Table 7). The lower the differences between the reference values and those predicted by the calibration models, the smaller the value of the standard error of prediction (SEP). Because of the wide range of samples analysed to provide adequate variability in the parameters evaluated, the variation range effect (measurement range or mean of this range) on the SEP value was removed by standardising the predictive accuracy of each calibration model using three statistical parameters (SEP\%, RPD and RPIQ). SEP\% values lower than 20 are considered acceptable for most analytical purposes (Cozzolino et al., 2008), and therefore also to determine FRV and PRO in intact berry seeds for wine grape varieties belonging to clusters 1 and 2, respectively.

The SEP value was better standardised by the residual predictive deviation (RPD) and residual predictive interquartile amplitude (RPIQ) indices (Table 7). When the SEP value is small when compared to the population spread of a certain chemical parameter, a relatively high index is obtained. Therefore, the higher the RPD value, the greater the predictive accuracy. Some authors have established standards indicating that RPD values higher than 2.0 correspond to very satisfactory calibration models for prediction purposes, whereas values ranging between 1.4 and 2.0 are indicative of fair models (Chang et al., 2001). More recently, other researchers have proposed the use of the RPIQ index to better assess the predictive accuracy of the calibration models (Cozzolino et al., 2011). According to this criterion, the extractable content of PRO in the seeds, expressed by seed weight, may be satisfactorily predicted from $\mathrm{E}_{\mathrm{s}}$ for wine grape varieties belonging to cluster 2 $\left(\right.$ RPIQ $>2$ ). The predictive ability of $\mathrm{W}_{\mathrm{s}}$ was unreliable for quantitative purposes, but acceptable for screening of the extractable content of TF expressed by grape weight, and of FRV expressed by seed weight (RPIQ $=1.59-1.64$ ) for wine grape varieties included in cluster 1 . The remaining chemical parameters could not be reliably predicted from the mechanical parameters evaluated using univariate calibration models.

In an attempt to improve the statistical parameters, multivariate calibration models were developed (Table 7). 
The results obtained in cluster 2 showed that the predictive accuracy was good for the determination of the extractable content of ECG expressed by seed weight $\left(R_{c}=0.836\right.$, SEC $\%=19.3$, RPIQ $=3.5)$, satisfactory for TF and TD expressed by grape weight and for PRO expressed by seed weight $\left(\mathrm{R}_{\mathrm{c}}=0.771-0.859\right.$, SEC $\%<20$, RPIQ $\left.=2.1-2.6\right)$, and unreliable for quantitative purposes but acceptable for screening of the FRV/PRO ratio, $\mathrm{A}_{280}$ expressed by grape and seed weight, as well as of FRV and TM expressed by grape weight $\left(\mathrm{R}_{\mathrm{c}}=0.621-0.765, \mathrm{SEC} \%<25\right.$, RPIQ $\left.=1.5-2.0\right)$. Taking into account the smaller number of samples in cluster 2 , the calibration models were also constructed using full cross-validation (leave-one-out splitting). In this case, the accuracy was satisfactory for the prediction of PRO and ECG expressed by seed weight $\left(\mathrm{R}_{\mathrm{c}}=0.794-0.840\right.$, SEC $\%<20$, RPIQ ca. 2.5), but acceptable only for screening of FRV/ PRO and TF expressed by seed weight $\left(\mathrm{R}_{\mathrm{c}}=0.578-0.736\right.$, $\mathrm{SEC} \%<12$, RPIQ ca. 1.5). Other improvements were also achieved for the prediction of the chemical parameters in the three clusters by means of multivariate calibration models if compared to univariate calibrations. However, some of the statistical parameters studied could hinder the predictive ability.
The relevance for the wine industry of a fast estimation of the extractable content of ECG, PRO, FRV and even the FRV/PRO ratio in grape seeds is supported by the impact of these determinations on the sensory characteristics of the seeds, like astringency and bitterness, which have great repercussions in the wine quality. In this work, galloylation was restricted to ECG because this compound was the only galloylated monomeric flavanol found in the seeds. It is well known that galloylation increases the astringency perceived (Ferrer-Gallego et al., 2010). The spectrophotometric index PRO is mainly associated with the concentration of high molecular weight proanthocyanidins, whereas the FRV index is strongly sensitive to the presence of monomeric flavanols and is partially related to low molecular weight flavanols (Peleg et al., 1999; Cheynier et al., 2006; Obreque-Slier et al., 2011). Furthermore, oligomeric flavanols represent the main phenolic fraction released from the intact seeds during winemaking. Therefore, the extractable contents of PRO and FRV are important factors determining astringency and bitterness, respectively. Astringency was the most appropriate sensory attribute for the assessment of grape seed quality, and the compression parameters of the seeds were likely correlated with perceived astringency, bitterness,

TABLE 7

Analytical performance of calibration models developed for the chemical parameters related to phenolic composition from the instrumental mechanical properties of seeds for varieties belonging to the clusters defined by seed hardness.

\begin{tabular}{|c|c|c|c|c|c|c|c|c|c|}
\hline Chemical parameter & $\begin{array}{c}\text { Mechanical } \\
\text { parameter }\end{array}$ & $\mathrm{R}_{\mathrm{c}}$ & SEC & SEC $\%$ & $\mathrm{R}_{\mathrm{v}}$ & SEP & SEP\% & RPD & RPIQ \\
\hline \multicolumn{10}{|c|}{ Cluster 1} \\
\hline FRV (mg/kg grape) & $\mathrm{E}$ & 0.507 & 461.05 & 28.5 & 0.577 & 488.23 & 30.5 & 1.20 & 1.25 \\
\hline TD (mg/kg grape) & $\mathrm{F}_{\mathrm{s}}$ & 0.502 & 20.20 & 35.5 & 0.519 & 20.13 & 36.4 & 1.17 & 1.08 \\
\hline $\mathrm{A}_{280}(1 / \mathrm{kg}$ grape $)$ & \multirow{9}{*}{$\mathrm{W}_{\mathrm{s}}$} & 0.625 & 8.82 & 22.6 & 0.670 & 9.05 & 23.0 & 1.35 & 0.99 \\
\hline TF (mg/kg grape) & & 0.618 & 629.71 & 20.9 & 0.578 & 659.76 & 21.9 & 1.22 & 1.59 \\
\hline FRV (mg/kg grape) & & 0.643 & 409.65 & 25.3 & 0.636 & 452.48 & 28.3 & 1.30 & 1.35 \\
\hline $\mathrm{EC}(\mathrm{mg} / \mathrm{kg}$ grape $)$ & & 0.500 & 41.13 & 46.5 & 0.579 & 41.25 & 47.1 & 1.22 & 1.18 \\
\hline ECG (mg/kg grape) & & 0.603 & 0.43 & 24.9 & 0.522 & 0.49 & 28.9 & 1.16 & 1.14 \\
\hline $\mathrm{B}_{1}(\mathrm{mg} / \mathrm{kg}$ grape $)$ & & 0.552 & 10.23 & 39.1 & 0.582 & 10.09 & 39.8 & 1.23 & 0.99 \\
\hline TM (mg/kg grape) & & 0.500 & 172.60 & 76.0 & 0.623 & 163.02 & 71.6 & 1.27 & 0.80 \\
\hline TD (mg/kg grape) & & 0.569 & 19.22 & 33.8 & 0.572 & 19.29 & 34.9 & 1.22 & 1.12 \\
\hline FRV (mg/g seed) & & 0.500 & 8.77 & 19.6 & 0.628 & 8.29 & 18.7 & 1.27 & 1.64 \\
\hline $\mathrm{A}_{280}(1 / \mathrm{kg}$ grape $)$ & \multirow{13}{*}{$\mathrm{F}_{\mathrm{s}}, \mathrm{W}_{\mathrm{s}}, \mathrm{E}_{\mathrm{s}}, \mathrm{DI}_{\mathrm{s}}$} & 0.642 & 8.66 & 22.2 & 0.666 & 9.09 & 23.1 & 1.34 & 0.99 \\
\hline TF (mg/kg grape) & & 0.630 & 622.35 & 20.6 & 0.576 & 664.25 & 22.1 & 1.21 & 1.58 \\
\hline PRO (mg/kg grape) & & 0.579 & 424.29 & 21.4 & 0.501 & 527.58 & 26.6 & 1.15 & 1.70 \\
\hline FRV (mg/kg grape) & & 0.653 & 405.08 & 25.0 & 0.633 & 454.55 & 28.4 & 1.29 & 1.34 \\
\hline $\mathrm{CA}(\mathrm{mg} / \mathrm{kg}$ grape $)$ & & 0.541 & 132.95 & 97.1 & 0.655 & 121.93 & 88.1 & 1.32 & 0.61 \\
\hline EC (mg/kg grape) & & 0.529 & 40.17 & 45.4 & 0.584 & 40.95 & 46.8 & 1.23 & 1.19 \\
\hline ECG (mg/kg grape) & & 0.621 & 0.43 & 24.5 & 0.517 & 0.50 & 29.4 & 1.14 & 1.12 \\
\hline $\mathrm{B}_{1}(\mathrm{mg} / \mathrm{kg}$ grape $)$ & & 0.555 & 10.20 & 39.0 & 0.583 & 10.09 & 39.8 & 1.23 & 0.99 \\
\hline $\mathrm{B}_{2}(\mathrm{mg} / \mathrm{kg}$ grape $)$ & & 0.511 & 11.32 & 36.9 & 0.502 & 10.71 & 35.8 & 1.15 & 1.52 \\
\hline TM (mg/kg grape) & & 0.557 & 164.97 & 72.6 & 0.653 & 156.97 & 69.0 & 1.32 & 0.83 \\
\hline TD (mg/kg grape) & & 0.575 & 19.12 & 33.6 & 0.581 & 19.10 & 34.6 & 1.23 & 1.14 \\
\hline FRV (mg/g seed) & & 0.534 & 8.53 & 19.0 & 0.631 & 8.19 & 18.4 & 1.29 & 1.66 \\
\hline TM (mg/g seed) & & 0.500 & 3.99 & 64.6 & 0.639 & 3.49 & 56.8 & 1.29 & 1.03 \\
\hline
\end{tabular}


TABLE 7 (CONTINUED)

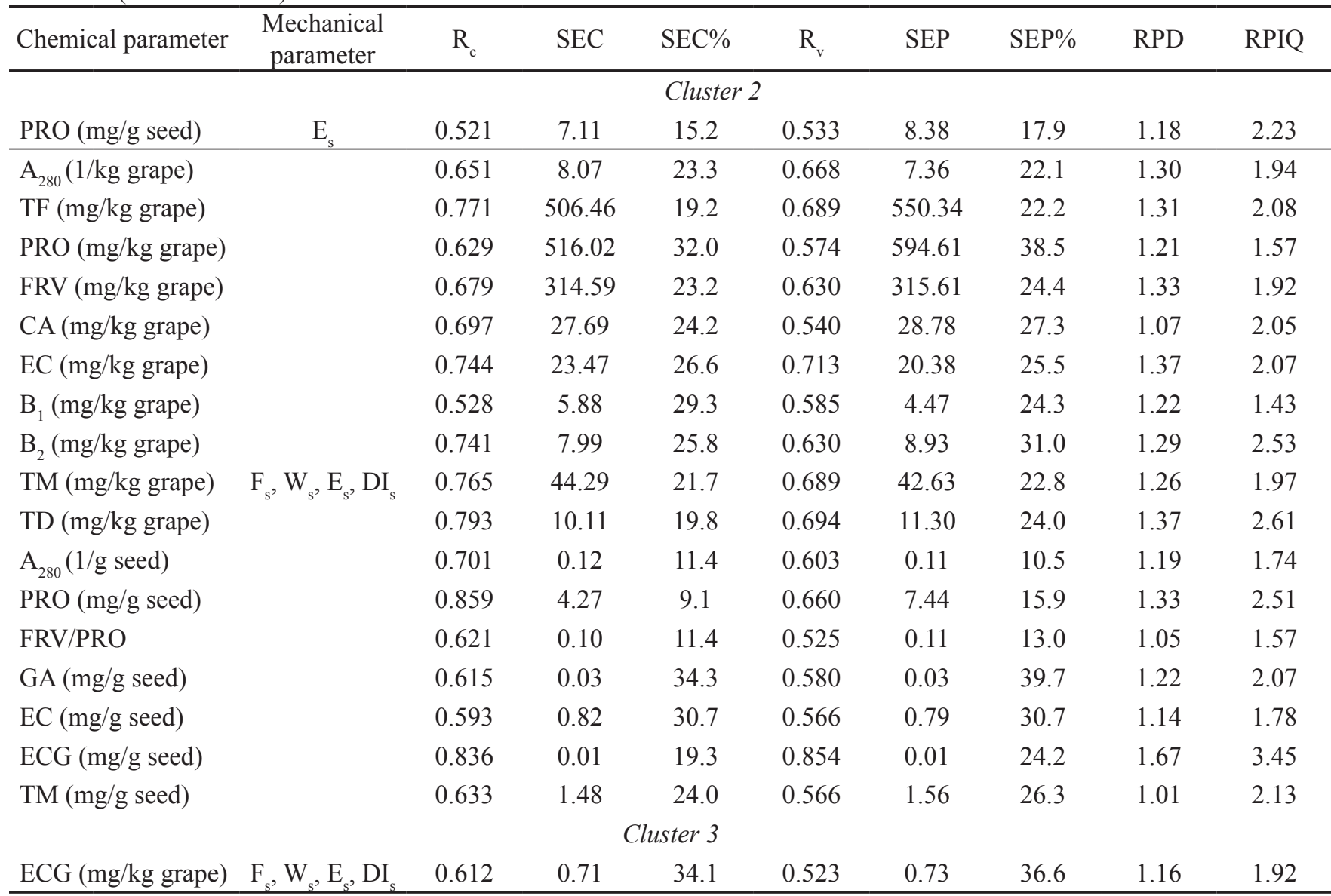

$\mathrm{n}=117$ for cluster $1, \mathrm{n}=45$ for cluster $2, \mathrm{n}=108$ for cluster $3 . \mathrm{R}=$ correlation coefficient of calibration, $\mathrm{SEC}=$ standard error of calibration, $\mathrm{SEC} \%=(\mathrm{SEC} / \mathrm{Mean}) \times 100, \mathrm{R}_{\mathrm{v}}=$ correlation coefficient of validation, $\mathrm{SEP}=$ standard error of prediction, $\mathrm{SEP} \%$ $=(\mathrm{SEP} /$ Mean $) \times 100, \mathrm{RPD}=$ residual predictive deviation $(\mathrm{SD} / \mathrm{SEP}), \mathrm{SD}=$ standard deviation, $\mathrm{RPIQ}=$ residual predictive interquartile amplitude (IQ/SEP), IQ = interquartile amplitude. $\mathrm{F}_{\mathrm{s}}=$ seed break force, $\mathrm{W}_{\mathrm{s}}=$ seed break energy, $\mathrm{E}_{\mathrm{s}}=$ resistance of the seed to the axial deformation, $\mathrm{DI}_{\mathrm{s}}=$ seed deformation index. $\mathrm{A}_{280}=$ absorbance measured at $280 \mathrm{~nm}, \mathrm{TF}=$ total flavonoids, $\mathrm{PRO}=$ proanthocyanidins, $\mathrm{FRV}=$ flavanols reactive to vanillin, $\mathrm{GA}=$ gallic acid, $\mathrm{CA}=$ catechin, $\mathrm{EC}=$ epicatechin, $\mathrm{ECG}=$ epicatechin gallate, $\mathrm{B}_{1}=$ procyanidin $\mathrm{B}_{1}, \mathrm{~B}_{2}=$ procyanidin $\mathrm{B}_{2}, \mathrm{TM}=$ total monomers, $\mathrm{TD}=$ total dimers.

vegetal aroma and roasted aroma (Letaief et al., 2013).

Regarding wine grape varieties belonging to cluster 3, no instrumental mechanical property may reliably predict any chemical parameter related to the phenolic composition of the seeds (Tables 6 and 7). At this point it was necessary to determine whether linear regression calibration models could be developed to predict the spectrophotometric indices $\left(\mathrm{A}_{280}, \mathrm{TF}, \mathrm{PRO}\right.$ and $\left.\mathrm{FRV}\right)$ with adequate reliability from direct instrumental measurement of the mechanical parameters of the seeds $\left(\mathrm{F}_{\mathrm{s}}, \mathrm{W}_{\mathrm{s}}, \mathrm{E}_{\mathrm{s}}\right.$ and $\left.\mathrm{DI}_{\mathrm{s}}\right)$ for some of the varieties belonging to cluster 3, but working with one cultivar individually, particularly Nebbiolo. A suitable variability in the reference values was assured by means of the analysis of seeds from grape berries sampled in different vineyards, at different ripening stages, during two consecutive years. In this case, only spectrophotometric indices were evaluated because they are more usually used in wineries to assess the phenolic maturity of the seeds, and were better correlated with the mechanical properties of the varieties in clusters 1 and 2. A correlation study carried out on Nebbiolo seeds showed that the highest significant correlation coefficients between the spectrophotometric indices and the instrumental mechanical parameters corresponded to $\mathrm{TF}$ with $\mathrm{F}_{\mathrm{s}}$ and
$\mathrm{W}_{\mathrm{s}}$, but the relationships found were weak $(\mathrm{R}=0.418$ $0.459, \mathrm{p}<0.001)$. This shortcoming was overcome using multivariate calibration models. Table 8 shows the mean and standard deviation values for each chemical and mechanical parameter, showing significant correlations between them $(p<0.01)$, as well as the statistical parameters of calibration and validation. The best analytical performance of the multivariate calibration models corresponded to the prediction of the chemical parameters expressed per grape weight. The ability of three mechanical attributes $\left(\mathrm{F}_{\mathrm{s}}, \mathrm{W}_{\mathrm{s}}\right.$ and $\left.\mathrm{E}_{\mathrm{s}}\right)$ to predict the extractable content of TF and PRO in the seeds, expressed per grape weight, was satisfactory, indicating an acceptable robustness of the multivariate calibration models for quantitative purposes $\left(\mathrm{R}_{\mathrm{c}}>0.68, \mathrm{SEC} \%\right.$ ca. $\left.20, \mathrm{RPIQ}>2.2\right)$. Nevertheless, the high SEC\% values found for the FRV/PRO ratio hindered the recommendation of the corresponding calibration model, even for screening purposes.

\section{CONCLUSIONS}

This study provides an interesting approach for understanding the associations between the instrumental mechanical properties and the phenolic composition of the 
TABLE 8

Analytical performance of multivariate calibration models developed for the spectrophotometric indices related to the phenolic composition of Nebbiolo seeds from the instrumental mechanical properties.

\begin{tabular}{|c|c|c|c|c|c|c|c|c|c|c|c|}
\hline $\begin{array}{l}\text { Chemical } \\
\text { parameter }\end{array}$ & Mean $\pm \mathrm{SD}^{\mathrm{a}}$ & $\begin{array}{l}\text { Mechanical } \\
\text { parameters }\end{array}$ & Mean $\pm \mathrm{SD}^{\mathrm{a}}$ & $\mathrm{R}_{\mathrm{c}}$ & SEC & SEC $\%$ & $\mathrm{R}_{\mathrm{v}}$ & SEP & SEP\% & RPD & RPIQ \\
\hline TF (mg/kg grape) & $2249 \pm 928$ & & & 0.858 & 483.48 & 21.6 & 0.886 & 424.41 & 18.6 & 2.14 & 3.95 \\
\hline PRO (mg/kg grape) & $903 \pm 273$ & $\mathrm{~F}_{\mathrm{s}}$ & $48.19 \pm 4.22$ & 0.682 & 203.00 & 22.3 & 0.665 & 199.61 & 22.5 & 1.33 & 2.24 \\
\hline $\mathrm{TF}$ (mg/g seed) & $58.7 \pm 22.7$ & $\mathrm{~W}_{\mathrm{s}}$ & $11.50 \pm 1.53$ & 0.782 & 14.43 & 24.6 & 0.798 & 13.43 & 22.9 & 1.65 & 2.79 \\
\hline PRO (mg/g seed) & $23.7 \pm 6.9$ & $\mathrm{E}_{\mathrm{s}}$ & $92.96 \pm 7.43$ & 0.534 & 5.93 & 24.7 & 0.501 & 5.93 & 25.9 & 1.15 & 1.85 \\
\hline FRV/PRO & $0.79 \pm 0.39$ & & & 0.713 & 0.24 & 31.4 & 0.674 & 0.34 & 41.8 & 1.34 & 1.56 \\
\hline
\end{tabular}

${ }^{a} n=136 . R_{c}=$ correlation coefficient of calibration, $\mathrm{SEC}=$ standard error of calibration, $\mathrm{SEC} \%=(\mathrm{SEC} / \mathrm{Mean}) \times 100, \mathrm{R}_{\mathrm{y}}=$ correlation coefficient of validation, $\mathrm{SEP}=$ standard error of prediction, $\mathrm{SEP} \%=(\mathrm{SEP} / \mathrm{Mean}) \times 100, \mathrm{RPD}=$ residual predictive deviation $(\mathrm{SD} / \mathrm{SEP}), \mathrm{SD}=$ standard deviation, $\mathrm{RPIQ}=$ residual predictive interquartile amplitude $(\mathrm{IQ} / \mathrm{SEP}), \mathrm{IQ}=\mathrm{interquartile}$ amplitude. $\mathrm{F}_{\mathrm{s}}=$ seed break force, $\mathrm{W}_{\mathrm{s}}=$ seed break energy, $\mathrm{E}_{\mathrm{s}}=$ resistance of the seed to the axial deformation. $\mathrm{TF}=$ total flavonoids, $\mathrm{PRO}=$ proanthocyanidins, $\mathrm{FRV}=$ flavanols reactive to vanillin.

seeds. At harvest, the berry heterogeneity in the vineyard had a small effect on the compression parameters of the seeds. Nevertheless, the seed hardness represents an important factor in the characterisation and differentiation of wine grape varieties. This varietal classification was useful in the assessment of the mechanical properties as possible predictors of the phenolic composition of the seeds. In fact, the robustness of the prediction for some spectrophotometric indices, as well as for monomeric and dimeric flavanols, was highly related to seed hardness. Among the calibration models developed, a few could be recommended for quantitative purposes (total flavonoids, proanthocyanidins, epicatechin gallate and total dimer flavanol content), while others were only acceptable for screening. The predictive accuracy could be improved for varieties with higher seed hardness, where the significant correlations between mechanical and chemical parameters were low, by constructing separate calibration models for each cultivar. Harvesting wine grapes at optimal seed maturity is one of the first steps in producing high quality wines and, in this sense, the knowledge of the mechanical properties of the seeds would enable rapid prediction of the extractable content of phenolic compounds affecting sensory characteristics.

\section{LITERATURE CITED}

Bellon-Maurel, V., Fernandez-Ahumada, E., Palagos, B., Roger, J.-M. \& McBratney, A., 2010. Critical review of chemometric indicators commonly used for assessing the quality of the prediction of soil attributes by NIR spectroscopy. Trends Anal. Chem. 29, 1073-1081.

Cáceres, A., Peña-Neira, A., Galvez, A., Obreque-Slier, E., López-Solís, R. \& Canals, J.M., 2012. Phenolic compositions of grapes and wines from cultivar Cabernet Sauvignon produced in Chile and their relationship to commercial value. J. Agric. Food Chem. 60, 8694-8702.

Cadot, Y., Miñana-Castelló, M.T. \& Chevalier, M., 2006. Anatomical, histological, and histochemical changes in grape seeds from Vitis vinifera L. cv Cabernet franc during fruit development. J. Agric. Food Chem. 54, 9206-9215.

Chang, C.-W., Laird, D.A., Mausbach, M.J. \& Hurburgh Jr., C.R., 2001. Near-infrared reflectance spectroscopy-principal components regression analyses of soil properties. Soil Sci. Soc. Am. J. 65, 480-490.
Cheynier, V., Dueñas-Paton, M., Salas, E., Maury, C., Souquet, J.M., Sarni-Manchado, P. \& Fulcrand, H., 2006. Structure and properties of wine pigments and tannins. Am. J. Enol. Vitic. 57, 298-305.

Cozzolino, D., Cynkar, W.U., Dambergs, R.G., Mercurio, M.D. \& Smith, P.A., 2008. Measurement of condensed tannins and dry matter in red grape homogenates using near infrared spectroscopy and partial least squares. J. Agric. Food Chem. 56, 7631-7636.

Cozzolino, D., Cynkar, W., Shah, N. \& Smith, P., 2011. Quantitative analysis of minerals and electric conductivity of red grape homogenates by near infrared reflectance spectroscopy. Comput. Electron. Agric. 77, 81-85.

Dai, Z.W., Genard, M., Li, S.H. \& Vivin, P., 2009. Analyzing the functional association among seed traits, berry growth and chemical composition in Cabernet-Sauvignon berry (Vitis vinifera L.) using a mathematical growth function. J. Int. Sci. Vigne Vin 43, 35-44.

Di Stefano, R. \& Cravero, M.C., 1991. Metodi per lo studio dei polifenoli dell'uva. Riv. Vitic. Enol. 44, 37-45.

Downey, M.O., Harvey, J.S. \& Robinson, S.P., 2003. Analysis of tannins in seeds and skins of Shiraz grapes throughout berry development. Aus. J. Grape Wine Res. 9, 15-27.

Ferrer-Gallego, R., García-Marino, M., Hernández-Hierro, J.M., RivasGonzalo, J.C. \& Escribano-Bailón, M.T., 2010. Statistical correlation between flavanolic composition, colour and sensorial parameters in grape seed during ripening. Anal. Chim. Acta 660, 22-28.

Giordano, M., Rolle, L., Zeppa, G. \& Gerbi, V., 2009. Chemical and volatile composition of three Italian sweet white Passito wines. J. Int. Sci. Vigne Vin 43, 159-170.

Kennedy, J.A., Matthews, M.A. \& Waterhouse, A.L., 2000a. Changes in grape seed polyphenols during fruit ripening. Phytochem. 55, 77-85.

Kennedy, J.A., Troup, G.J., Pilbrow, J.R., Hutton, D.R., Hewitt, D., Hunter, C.R., Ristic, R., Iland, P.G. \& Jones, G.P., 2000b. Development of seed polyphenols in berries from Vitis vinifera L. cv. Shiraz. Aus. J. Grape Wine Res. 6, 244-254.

Kotseridis, Y., Georgiadou, A., Tikos, P., Kallithraka, S. \& Koundouras, S., 2012. Effects of severity of post-flowering leaf removal on berry growth and composition of three red Vitis vinifera L. cultivars grown under semiarid conditions. J. Agric. Food Chem. 60, 6000-6010.

Le Moigne, M., Maury, C., Bertrand, D. \& Jourjon, F., 2008. Sensory and instrumental characterisation of Cabernet Franc grapes according to ripening stages and growing location. Food Qual. Pref. 19, 220-231. 
Letaief, H., Maury, C., Symoneaux, R. \& Siret, R., 2013. Sensory and instrumental texture measurements for assessing grape seed parameters during fruit development. J. Sci. Food Agric. 93(10), 2531-2540.

Lorrain, B., Chira, K. \& Teissedre, P.-L., 2011. Phenolic composition of Merlot and Cabernet-Sauvignon grapes from Bordeaux vineyard for the 2009-vintage: Comparison to 2006, 2007 and 2008 vintages. Food Chem. 126, 1991-1999.

Mattivi, F., Vrhovsek, U., Masuero, D. \& Trainotti, D., 2009. Differences in the amount and structure of extractable skin and seed tannins amongst red grape varieties. Aus. J. Grape Wine Res. 15, 27-35.

McRae, J.M. \& Kennedy, J.A., 2011. Wine and grape tannin interactions with salivary proteins and their impact on astringency: A review of current research. Molecules 16, 2348-2364.

Monagas, M., Gómez-Cordovés, C., Bartolomé, B., Laureano, O. \& Ricardo da Silva, J.M., 2003. Monomeric, oligomeric, and polymeric flavan-3ol composition of wines and grapes from Vitis vinifera L. cv. Graciano, Tempranillo, and Cabernet Sauvignon. J. Agric. Food Chem. 51, 6475-6481.

Noguerol-Pato, R., González-Barreiro, C., Cancho-Grande, B., Santiago, J.L., Martínez, M.C. \& Simal-Gándara, J., 2012. Aroma potential of Brancellao grapes from different cluster positions. Food Chem. 132, 112124

Obreque-Slier, E., López-Solís, R., Castro-Ulloa, L., Romero-Díaz, C. \& Peña-Neira, A., 2012. Phenolic composition and physicochemical parameters of Carménère, Cabernet Sauvignon, Merlot and Cabernet Franc grape seeds (Vitis vinifera L.) during ripening. LWT - Food Sci. Technol. $48,134-141$

Obreque-Slier, E., Peña-Neira, A. \& López-Solís, R., 2011. Precipitation of low molecular weight phenolic compounds of grape seeds cv. Carménère (Vitis vinifera L.) by whole saliva. Eur. Food Res. Technol. 232, 113-121.

OIV, 2008. Recueil international des méthodes d'analyse des vins et des moûts. OIV, Paris.

Peleg, H., Gacon, K., Schlich, P. \& Noble, A.C., 1999. Bitterness and astringency of flavan-3-ol monomers, dimers and trimers. J. Sci. Food Agric. 79, 1123-1128.

Pérez-Magariño, S. \& González-San José, M.L., 2006. Polyphenols and colour variability of red wines made from grapes harvested at different ripeness grade. Food Chem. 96, 197-208.

Puškaš, V. \& Miljić, U., 2012. The application of D-optimal design for modelling the red wine ageing process. Food Control 28, 362-367.
Rodríguez Montealegre, R., Romero Peces, R., Chacón Vozmediano, J.L., Martínez Gascueña, J. \& García Romero, E., 2006. Phenolic compounds in skins and seeds of ten grape Vitis vinifera varieties grown in a warm climate. J. Food Comp. Anal. 19, 687-693.

Rolle, L., Giacosa, S., Torchio, F. \& Río Segade, S., 2012d. Changes in acoustic and mechanical properties of Cabernet Sauvignon seeds during ripening. Am. J. Enol. Vitic. 63, 413-418.

Rolle, L., Río Segade, S., Torchio, F., Giacosa, S., Cagnasso, E., Marengo, F. \& Gerbi, V., 2011. Influence of grape density and harvest date on changes in phenolic composition, phenol extractability indices, and instrumental texture properties during ripening. J. Agric. Food Chem. 59, 8796-8805.

Rolle, L., Siret, R., Río Segade, S., Maury, C., Gerbi, V. \& Jourjon, F., 2012c. Instrumental texture analysis parameters as markers of table-grape and winegrape quality: A review. Am. J. Enol. Vitic. 63, 11-28.

Rolle, L., Torchio, F., Giacosa, S. \& Gerbi, V., 2009. Modifications of mechanical characteristics and phenolic composition in berry skins and seeds of Mondeuse winegrapes throughout the on-vine drying process. J. Sci. Food Agric. 89, 1973-1980.

Rolle, L., Torchio, F., Giacosa, S., Río Segade, S., Cagnasso, E. \& Gerbi, V., 2012b. Assessment of physicochemical differences in Nebbiolo grape berries from different production areas and sorted by flotation. Am. J. Enol Vitic. 63, 195-204.

Rolle, L., Torchio, F., Lorrain, B., Giacosa, S., Río Segade, S., Cagnasso, E., Gerbi, V. \& Teissedre, P.-L., 2012a. Rapid methods for the evaluation of total phenol content and extractability in intact grape seeds of CabernetSauvignon: Instrumental mechanical properties and FT-NIR spectrum. J. Int. Sci. Vigne Vin 46, 29-40.

Sacchi, K.L., Bisson, L.F. \& Adams, D.O., 2005. A review of the effect of winemaking techniques on phenolic extraction in red wines. Am. J. Enol. Vitic. 56, 197-206.

Torchio, F., Cagnasso, E., Gerbi, V. \& Rolle, L., 2010. Mechanica properties, phenolic composition and extractability indices of Barbera grapes of different soluble solids contents from several growing areas. Anal. Chim. Acta 660, 183-189.

Torchio, F., Giacosa, S., Río Segade, S., Mattivi, F., Gerbi, V. \& Rolle, L., 2012. Optimization of a method based on the simultaneous measurement of acoustic and mechanical properties of winegrape seeds for the determination of the ripening stage. J. Agric. Food Chem. 60, 9006-9016 